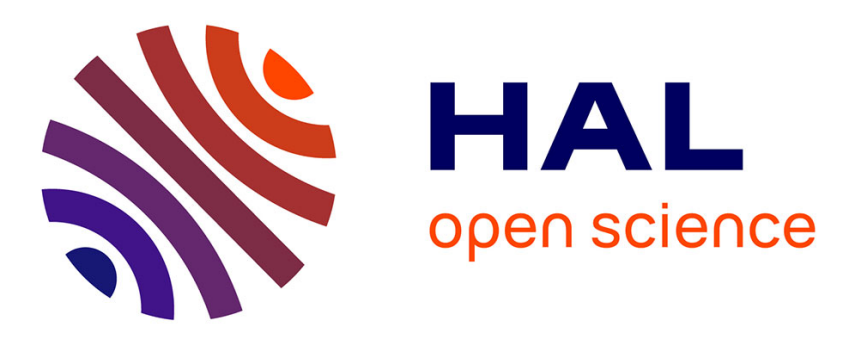

\title{
A higher order elasto-viscoplastic model using fast Fourier transforms: Effects of lattice curvatures on mechanical response of nanocrystalline metals
}

M.V. V Upadhyay, L. Capolungo, V. Taupin, Claude Fressengeas, R.A. A Lebensohn

\section{To cite this version:}

M.V. V Upadhyay, L. Capolungo, V. Taupin, Claude Fressengeas, R.A. A Lebensohn. A higher order elasto-viscoplastic model using fast Fourier transforms: Effects of lattice curvatures on mechanical response of nanocrystalline metals. International Journal of Plasticity, 2016, 83, pp.126-152. 10.1016/j.ijplas.2016.04.007 . hal-02943011

\section{HAL Id: hal-02943011 \\ https://hal.univ-lorraine.fr/hal-02943011}

Submitted on 18 Sep 2020

HAL is a multi-disciplinary open access archive for the deposit and dissemination of scientific research documents, whether they are published or not. The documents may come from teaching and research institutions in France or abroad, or from public or private research centers.
L'archive ouverte pluridisciplinaire HAL, est destinée au dépôt et à la diffusion de documents scientifiques de niveau recherche, publiés ou non, émanant des établissements d'enseignement et de recherche français ou étrangers, des laboratoires publics ou privés. 


\title{
A higher order elasto-viscoplastic model using fast Fourier transforms: effects of lattice curvatures on mechanical response of nanocrystalline metals
}

\author{
${ }^{a b 12}$ M. V. Upadhyay, ${ }^{a b}$ L. Capolungo, ${ }^{c}$ V. Taupin, ${ }^{c}$ C. Fressengeas, ${ }^{d}$ R. A. \\ Lebensohn \\ ${ }^{a}$ G.W. Woodruff School of Mechanical Engineering, Georgia Institute of Technology, \\ Atlanta 30332, Georgia, USA \\ ${ }^{b}$ Georgia Tech Lorraine/CNRS, 57070 Metz Cedex, France \\ ${ }^{c}$ Laboratoire d'Etude des Microstructures et de Mécanique des Matériaux, \\ Université de Lorraine/CNRS, Ile du Saulcy, 57045 Metz Cedex, France \\ ${ }^{d}$ Materials Science and Technology Division, \\ Los Alamos National Laboratory, MS G755, Los Alamos, NM 87845, USA
}

\begin{abstract}
In this work a couple stress continuum based elasto-viscoplastic fast Fourier transform model is developed with the intent to study the role of curvatures - gradient of rotation - on the local meso scale and effective macroscale mechanical response of nanocrystalline materials. Development of this model has led to the formulation of an extended periodic Lippmann Schwinger equation that accounts for couple stress equilibrium. In addition to the standard boundary conditions on strain rate and Cauchy stresses, the model allows imposing non-standard couple stress and curvature rate boundary conditions. Application to representative nanocrystalline microstructures reveals that elastic and plastic curvatures accommodate a part of the local and macroscopic Cauchy stresses. Next, grain boundary interfaces are characterized using curvatures that are representative of their structure and defect content. Depending on the magnitude and distribution of these curvatures, local stresses in the grain boundary neighborhood are generated that activate slip systems besides those fulfilling the Schmid criterion. Generation of both polar dislocations and disclinations as a possible plasticity mechanism
\end{abstract}

\footnotetext{
${ }^{1}$ corresponding author: manas.upadhyay@psi.ch

${ }^{2}$ Present address: WBBA 113, Paul Scherrer Institute, 5232 Villigen PSI, Switzerland.
} 
in nanocrystalline materials is explored. At the macro scale, this results in a strain rate dependent "softening" or the inverse Hall-Petch effect. The modeling framework naturally captures this grain size effect without any ad hoc assumptions.

Keywords: Polycrystalline material; grain boundaries; dislocations; curvatures; stress relaxation

\section{Introduction}

Plasticity in nanocrystalline (nc) metals and alloys occurs via an interplay between line crystal defects i.e. dislocations and disclinations, and grain boundary (GB) mechanisms. The dynamics of these interactions is dependent on the local microstructure which for a typical nc material is composed of a large volume fraction of heterogeneously distributed low and high angle GB interfaces [1,2]. Molecular static simulations have indicated that the structure of these GB interfaces determines their energy [3-5] which in conjunction with other morphological and textural aspects such as grain size and orientation distribution, plays a major role in defining the energy landscape of nc materials. These provide the driving forces for various non-conventional plasticity mechanisms [6, 7] resulting in the typical nc macro scale mechanical properties such as improved strength (Hall-Petch effect [8, 9]) and increased strain rate sensitivity $[10,11]$ in comparison with their coarse grained counterparts $[6,7]$. Furthermore, below a critical grain size some nc materials exhibit an inverse Hall-Petch or "softnening" effect where the material strength decreases [12-14].

Modeling the mechanical response of polycrystalline materials is traditionally pursued using classical continuum based meso-scale crystal plasticity models. Applications to nc materials have generally aimed at demonstrating the grain size effect on the macroscopic response based either on phenomenological assumptions of mechanical behavior of GBs and grain interiors [15-21], GB evolution laws derived from GB mechanisms [22, 23], or an elasto-viscoplastic response of GBs [24-28]. As a consequence of their size independent classical continuum framework, these models are unable to appropriately capture the underlying GB structure. Therefore, these models require introducing an ad hoc length scale parameter (typically the grain size) to capture the grain size dependent response. 
This problem can be alleviated with the help of distortion gradient plasticity models which intrinsically account for a length scale. These include models that account for the role of strain gradients and rotational gradients i.e. curvatures. While the role of strain gradients on plasticity in nc materials [29-37] has received considerable interest over the past decade, the role of rotational gradients i.e. lattice curvatures has received disproportionately less attention. The origin of this imbalance may lie in the fact that only recently experimental characterization techniques have achieved the resolutions to quantify lattice curvatures in nc materials [38-40]. These studies have revealed that lattice curvatures manifest themselves at locations of GB interfaces, triple junctions, defect cell structures, activation of different slip systems and heterogeneity in slip distribution and intensity on the same slip system. A series of continuum based studies at the nano scale on GBs [41] have highlighted that lattice curvatures are necessary to describe GB structure $[42,43]$ in order to obtain energy estimates that match those obtained from experiments and molecular statics simulations [44] and to model the structure sensitive non-local GB dynamics, for instance shear coupled boundary migration [45].

Deriving motivation from these studies, Taupin et al. [46] recently proposed a strain and curvature based multi-scale crystal plasticity model to study the role of incompatibilities in lattice curvature and strains on the mechanical response of nc materials. The model, known as phenomenological field dislocation and disclination mechanics (PMFDDM), accounts for the nucleation and dynamics of polar and statistical dislocations and disclinations. It is numerically implemented using a Galerkin-least square finite element approach [46] to study the non-local and size dependent meso scale GB dynamics in bi- and tri- crystalline structures [46].

Modeling representative volume elements (RVEs) of nc materials, however, is difficult to achieve using a finite element based approach; capturing the intricate details associated with GBs that occupy a large volume fraction of nc materials requires employing very fine meshes resulting in a very large stiffness matrix. This, coupled with the numerical complexity associated with solving the finite element problem, motivates the alternative use of fast Fourier transform (FFT) technique. The full field FFT based numerical approach for periodic RVEs was originally developed in a classical continuum framework for composites $[47,48]$ and later extended to viscoplastic [49] and elasto-viscoplastic [50] materials. Its advantage is in solving the local problem with a time complexity of $O(N \log N)$. 
In light of the above, the main objectives of this work are (a) to develop a numerical implementation of the PMFDDM model using the FFT technique and (b) apply to RVEs of nc materials to understand the role of lattice curvatures on their local and effective mechanical response. In order to facilitate part (b), only the meso scale component of the PMFDDM i.e. evolution of statistical dislocations and elasticity - will be considered. The resulting model is a couple stress continuum based elasto-viscoplastic (CSEVP) model. A couple stress based Green's function is proposed to analytically solve the CSEVP problem. A combined continuum and discrete FFT approach is developed for application to nc RVEs. The CSEVP FFT model allows characterizing initial microstructures using curvatures. This is developed and explored in detail. The role of using a curvature based approach on the local and bulk mechanical response of nc materials is then studied. As will be demonstrated later, characterizing the initial microstructure using curvatures captures the grain size dependent macroscopic strain rate sensitivity and "softening" effect often observed in nc materials.

The paper is divided into sections as follows. Section 2 describes the notations used throughout this work. In section 3, the governing equations of the CSEVP model are presented along with the technique used to characterize initial microstructures using lattice curvatures. Section 4 develops the FFT implementation of the CSEVP model. The numerical implementation is achieved using a discrete Fourier transform (DFT) technique. Section 5 describes the microstructure, elastic plastic properties and test conditions to study the role of curvatures. In section 6, the CSEVP DFT model is first benchmarked with respect to the EVP FFT model [50] for nc microstructures. Then, the role of initial lattice curvatures on the local and effective response of nc aggregates is studied. Section 7 provides a perspective on the predictive capabilities of the CSEVP DFT model. Main conclusions of this study are summarized in section 8 followed by references and appendix.

\section{Notations}

The following notations are used everywhere in this paper. A bold symbol denotes a tensor. When there may be ambiguity, an arrow is superposed to represent a vector: $\overrightarrow{\mathbf{V}}$. The symmetric part of tensor $\mathbf{A}$ is denoted $\mathbf{A}^{s}$. Its skew-symmetric or anti-symmetric part is $\mathbf{A}^{a}$. The tensor $\mathbf{A} . \mathbf{B}$, with rectangular Cartesian components $A_{i k} B_{k j}$, results from the dot product of tensors $\mathbf{A}$ and $\mathbf{B}$, and $\mathbf{A} \otimes \mathbf{B}$ is their tensorial product, with components 
$A_{i j} B_{k l}$. A : represents the trace inner product of the two second order tensors $\mathbf{A}: \mathbf{B}=A_{i j} B_{i j}$, in rectangular Cartesian components, or the product of a higher order tensor with a second order tensor, e.g., $\mathbf{A}: \mathbf{B}=A_{i j k l} B_{k l}$. The cross product of a second-order tensor $\mathbf{A}$ and a vector $\mathbf{V}$, the $\operatorname{div}$ and curl operations for second-order tensors are defined row by row, in analogy with the vectorial case. For any base vector $\mathbf{e}_{i}$ of the reference frame:

$$
\begin{aligned}
(\mathbf{A} \times \mathbf{V})^{t} \cdot \mathbf{e}_{i} & =\left(\mathbf{A}^{t} \cdot \mathbf{e}_{i}\right) \times \mathbf{V} \\
(\operatorname{div} \mathbf{A})^{t} \cdot \mathbf{e}_{i} & =\operatorname{div}\left(\mathbf{A}^{t} \cdot \mathbf{e}_{i}\right) \\
(\operatorname{curl} \mathbf{A})^{t} \cdot \mathbf{e}_{i} & =\operatorname{curl}\left(\mathbf{A}^{t} \cdot \mathbf{e}_{i}\right) .
\end{aligned}
$$

In rectangular Cartesian components:

$$
\begin{aligned}
(\mathbf{A} \times \mathbf{V})_{i j} & =e_{j k l} A_{i k} V_{l} \\
(\operatorname{div} \mathbf{A})_{i} & =A_{i j, j} \\
(\operatorname{curl} \mathbf{A})_{i j} & =e_{j k l} A_{i l, k} .
\end{aligned}
$$

where $e_{j k l}$ is a component of the third-order alternating Levi-Civita tensor $\mathbf{X}$. A vector $\overrightarrow{\mathbf{A}}$ is associated with tensor $\mathbf{A}$ by using its trace inner product with tensor $\mathbf{X}$ :

$$
(\overrightarrow{\mathbf{A}})_{k}=-\frac{1}{2}(\mathbf{A}: \mathbf{X})_{k}=-\frac{1}{2} e_{i j k} A_{i j} .
$$

In the component representation, the spatial derivative with respect to a Cartesian coordinate is indicated by a comma followed by the component index. A superposed dot represents a material time derivative.

\section{A couple stress elasto-viscoplastic model}

In this section, the statistical component of the PMFDDM model, i.e. CSEVP model, is briefly recalled. For the complete PMFDDM model one is directed towards the work of Taupin et al. [46].

\subsection{Kinematics}

A polycrystalline crack/void free material (volume $V$ ) is treated as a heterogeneous body containing an arbitrary distribution of line crystal defects

- dislocations and disclinations - within the bulk of each granular domain and at GB interfaces. 
The total displacement field $\overrightarrow{\boldsymbol{u}}$ is continuously defined everywhere in the domain. This allows for the definition of the total distortion field $\boldsymbol{U}=$ $\operatorname{grad} \overrightarrow{\boldsymbol{u}}$ as the gradient of the displacement, giving the compatibility condition curl $\boldsymbol{U}=0$. The symmetric and anti-symmetric components of the compatible (curl free) total distortion $(\boldsymbol{U})$ are the compatible total strain $\boldsymbol{\epsilon}$ and rotation $\boldsymbol{\omega}$ tensors, respectively. The rotation tensor can be represented in the form of a vector as $\overrightarrow{\boldsymbol{\omega}}=-\frac{1}{2} \boldsymbol{\omega}: \mathbf{X}$ which is related to the total displacement as $\overrightarrow{\boldsymbol{\omega}}=\frac{1}{2} \operatorname{curl} \overrightarrow{\boldsymbol{u}}$. The gradient of total rotation vector is the compatible total curvature $\boldsymbol{\kappa}=\operatorname{grad} \overrightarrow{\boldsymbol{\omega}}$.

In presence of an ensemble of dislocations and disclinations, the total strain and curvature can be decomposed into elastic and plastic components as,

$$
\begin{aligned}
\epsilon & =\epsilon^{e}+\epsilon^{p} \\
\kappa & =\kappa^{e}+\kappa^{p}
\end{aligned}
$$

where the superscripts ' $e$ ' and ' $p$ ' correspond to the elastic and plastic components, respectively.

The polar dislocation $(\boldsymbol{\alpha})$ and disclination $(\boldsymbol{\theta})$ densities are defined as $[51-54]$,

$$
\begin{gathered}
\boldsymbol{\alpha}=\operatorname{curl} \epsilon^{e}-\boldsymbol{\kappa}^{e \boldsymbol{T}}+\operatorname{tr}\left(\boldsymbol{\kappa}^{e}\right) \boldsymbol{I}=-\operatorname{curl} \epsilon^{p}+\boldsymbol{\kappa}^{p \boldsymbol{T}}-\operatorname{tr}\left(\boldsymbol{\kappa}^{p}\right) \boldsymbol{I} \\
\boldsymbol{\theta}=\operatorname{curl} \boldsymbol{\kappa}^{e}=-\operatorname{curl} \boldsymbol{\kappa}^{p}
\end{gathered}
$$

Note that in the work of Nye [55], the dislocation density tensor is obtained by neglecting the curl of the elastic/plastic strain field in equation (10).

\subsection{Elasticity}

During elastic deformation under the action of traction $(\overrightarrow{\boldsymbol{t}})$ and moment $(\overrightarrow{\boldsymbol{m}})$ boundary conditions (on surface $\boldsymbol{S}$ ), asymmetric force $(\boldsymbol{\sigma})$ and couple $(\boldsymbol{M})$ stresses are generated.

$$
\begin{aligned}
\overrightarrow{\boldsymbol{t}} & =\boldsymbol{\sigma} \cdot \overrightarrow{\boldsymbol{n}} \\
\overrightarrow{\boldsymbol{m}} & =M \cdot \overrightarrow{\boldsymbol{n}}
\end{aligned}
$$

where $\overrightarrow{\boldsymbol{n}}$ is the normal to the $\boldsymbol{S}$. In the absence of body forces and couples, the higher order equilibrium equation [56] is:

$$
\operatorname{div} \boldsymbol{\sigma}^{s}+\frac{1}{2} \operatorname{div} \operatorname{curl} \boldsymbol{M}^{D}=0 \quad \text { or } \quad \sigma_{i j, j}^{s}+\frac{1}{2} e_{i j k} M_{k l, l j}^{D}=0
$$


where the superscripts $s$ and $D$ correspond to the symmetric and deviatoric components, respectively.

Assuming centrosymmetry everywhere in the domain, the Helmholtz free energy density is defined as [57]: $\psi=\frac{1}{2} \boldsymbol{\epsilon}^{e}: \boldsymbol{C}: \boldsymbol{\epsilon}^{e}+\frac{1}{2} \boldsymbol{\kappa}^{e}: \boldsymbol{A}: \boldsymbol{\kappa}^{e}$; where $\boldsymbol{A}$ and $\boldsymbol{C}$ are elasticity tensors. The Cauchy and couple stresses are defined as [56-58],

$$
\begin{array}{r}
\boldsymbol{\sigma}^{s}=\boldsymbol{C}: \overline{\boldsymbol{\epsilon}}^{e} \\
\boldsymbol{M}^{D}=\boldsymbol{A}: \overline{\boldsymbol{\kappa}}^{e}
\end{array}
$$

Elastic constant $\boldsymbol{C}$ is the well-known stiffness tensor from classical elasticity. Elastic constant $\boldsymbol{A}$ has a magnitude of the order $G$ (shear modulus) $\times$ $\left(\mathrm{r}^{2}\right) ; r$ is a length scale measure. In the literature, its estimates are limited and sometimes differ by an order of 10 in magnitude. Kröner [59] proposed a magnitude of $A=G b^{2}$ at the length scale of individual dislocations, where $b$ is the Burgers vector magnitude. The meaning of Kröners choice is that the Burgers vector magnitude is a plausible characteristic length scale for the extent of areas where non-local effects arising from the presence of dislocations are significant. Whereas, in recent works, a length scale of $0.05 \mathrm{~nm}$ was proposed for Copper [60] and 0.3 times the lattice spacing for $\alpha$-iron [61]. Taupin et al. [46] proposed a multi-scale law for the magnitude of $\boldsymbol{A}$. In the present work, Kröner's estimate of $A=G b^{2}$ is used to highlight the importance of curvatures.

\subsection{Plasticity}

Now let the heterogeneous crack/void free body undergo plastic deformation under the action of traction and moment boundary conditions. This results in the evolution of statistical and geometrically necessary ensembles of defects. From a meso scale perspective, this contributes to the evolution of plastic strain and curvature rates. In the present work the motion of geometrically necessary ensembles of line crystal defects - described with the help of transport equations [46, 62] - and statistical ensemble of disclinations [46] - described with the help of couple stress based plastic curvature rate is neglected. Evolution of statistical ensembles of dislocations is described using the statistical plastic slip rate tensor $\left(\boldsymbol{L}_{\boldsymbol{p}}\right)$ as,

$$
\boldsymbol{L}_{\boldsymbol{p}}=\sum_{\alpha} \dot{\gamma}_{\alpha}^{p} \boldsymbol{m}_{\alpha}=\sum_{\alpha} \boldsymbol{m}_{\alpha} \dot{\gamma}_{0}^{p}\left(\frac{\left|\boldsymbol{\sigma}^{s}: \boldsymbol{m}_{\alpha}\right|}{\tau_{\alpha}}\right)^{n} \operatorname{sgn}\left(\boldsymbol{\sigma}^{s}: \boldsymbol{m}_{\alpha}\right)
$$


where the subscript $\alpha$ represents the slip system, $\tau_{\alpha}$ is the critical resolved shear stress in the slip system $\alpha, n$ is the exponent for the plastic slip rate. $\boldsymbol{m}=\overrightarrow{\boldsymbol{b}}_{\alpha} \otimes \overrightarrow{\boldsymbol{n}}_{\alpha}$ is the Schmid tensor where $\overrightarrow{\boldsymbol{b}}_{\alpha}$ and $\overrightarrow{\boldsymbol{n}}_{\alpha}$ are respectively the Burgers vector and normal to the slip plane for slip system $\alpha$. The plastic strain rate $\dot{\boldsymbol{\epsilon}}^{p}$ is defined as the symmetric part of the plastic slip rate

$$
\dot{\boldsymbol{\epsilon}}^{p}=\boldsymbol{L}_{\boldsymbol{p}}^{s}=\sum_{\alpha} \dot{\gamma}_{\alpha}^{p} \boldsymbol{P}_{\alpha}
$$

$\boldsymbol{P}_{\alpha}=\frac{1}{2}\left(\overrightarrow{\boldsymbol{b}}_{\alpha} \otimes \overrightarrow{\boldsymbol{n}}_{\alpha}+\overrightarrow{\boldsymbol{n}}_{\alpha} \otimes \overrightarrow{\boldsymbol{b}}_{\alpha}\right)$ is the symmetric slip orientation tensor.

Temporal evolution of statistical dislocations results in a local change in the plastic curvatures. The plastic curvature rate $\left(\dot{\boldsymbol{\kappa}}^{p}\right)$ is given as,

$$
\dot{\boldsymbol{\kappa}}^{p}=-\frac{1}{2} \operatorname{grad}\left(\boldsymbol{L}_{\boldsymbol{p}}{ }^{a}: \mathrm{X}\right)=-\frac{\mathbf{1}}{\mathbf{2}} \operatorname{grad}\left(\sum_{\alpha} \dot{\gamma}_{\alpha}^{\mathrm{p}} \boldsymbol{Q}_{\alpha}: \mathbf{X}\right)
$$

where $\boldsymbol{Q}_{\alpha}$ is the anti-symmetric orientation tensor $\frac{1}{2}\left(\overrightarrow{\boldsymbol{b}}_{\alpha} \otimes \overrightarrow{\boldsymbol{n}}_{\alpha}-\overrightarrow{\boldsymbol{n}}_{\alpha} \otimes \overrightarrow{\boldsymbol{b}}_{\alpha}\right)$ for slip system $\alpha$ and $\mathbf{X}$ is the third order Levi-Civita permutation tensor. Plastic curvature rates similar to equation (19) have been proposed in the dislocation based Cosserat [63] and micropolar [64] single crystal plasticity theories.

\subsection{Characterizing GB interface using curvatures}

The classical continuum approach to study polycrystalline materials is capable of capturing the differences in elastic and plastic properties of each grain and their mismatch at the GB interface. With the couple stress continuum based approach it is possible to capture a part of the initial microstructure by explicitly accounting for the GB misorientation, plane orientation, its symmetric/asymmetric nature, and tilt/twist nature. This is done by characterizing GBs using initial curvature fields $\kappa_{i j}^{i n i}$.

As a first attempt to define $\kappa_{i j}^{i n i}$, these are taken to be equal to the ratio of the variation of rigid body rotation $\Delta \omega_{i}^{i n i}$ over a distance $\Delta x_{j}$ across the boundary:

$$
\kappa_{i j}^{i n i}=\frac{\Delta \omega_{i}^{i n i}}{\Delta x_{j}}
$$

Here $\Delta \omega_{i}^{i n i}$ would depend on the difference in initial rigid body rotations of the grains across the boundary while the magnitude of $\Delta x_{j}$ would correspond to the mesh resolution. This first approximation is in accordance 
with the definition of compatible curvatures as the gradient of rotation vector defined in section 3.1.

\section{Fourier transform based couple stress elasto-viscoplastic formu- lation for heterogeneous materials}

\subsection{An extended periodic Lippmann Schwinger formulation for couple stress media}

In the pioneering work of Moulinec and Suquet [47, 48], a classical continuum framework was adopted to obtain the effective response of a composite material along a path defined by macroscopic Cauchy stresses and strains by solving the local problem in an RVE. In this work, the classical framework is extended to account for couple stress continuum. Since this is a new development, it is described in detail in the following.

It is assumed that all grains are perfectly bonded i.e. displacements, tractions and moments are continuous across GB interfaces. Periodic boundary conditions are imposed along the microstructure reference directions. Let the total displacement field $(\overrightarrow{\boldsymbol{u}}(\overrightarrow{\boldsymbol{x}}))$ be split into a mean field $(\overrightarrow{\boldsymbol{U}})$ and a fluctuation field $\tilde{\boldsymbol{u}}(\overrightarrow{\boldsymbol{x}})$. The total strain $(\boldsymbol{\epsilon}(\overrightarrow{\boldsymbol{x}}))$ and curvature $(\boldsymbol{\kappa}(\overrightarrow{\boldsymbol{x}}))$ fields can also be split into their volume averages $\boldsymbol{E}$ and $\boldsymbol{K}$ along with fluctuations in local fields $\tilde{\boldsymbol{\epsilon}}(\overrightarrow{\boldsymbol{x}})$ and $\tilde{\boldsymbol{\kappa}}(\overrightarrow{\boldsymbol{x}})$, respectively.

$$
\begin{array}{r}
\boldsymbol{\epsilon}(\overrightarrow{\boldsymbol{x}})=\boldsymbol{E}+\tilde{\boldsymbol{\epsilon}}(\overrightarrow{\boldsymbol{x}}) \\
\boldsymbol{\kappa}(\overrightarrow{\boldsymbol{x}})=\boldsymbol{K}+\tilde{\boldsymbol{\kappa}}(\overrightarrow{\boldsymbol{x}})
\end{array}
$$

Periodic boundary conditions imply that the fluctuating displacement $\tilde{\boldsymbol{u}}(\overrightarrow{\boldsymbol{x}})$, is periodic on parallel surfaces. In order to satisfy the equilibrium equations the traction and moment vectors i.e. $\boldsymbol{\sigma} \cdot \overrightarrow{\boldsymbol{n}}$ and $\boldsymbol{M} \cdot \overrightarrow{\boldsymbol{n}}$, respectively, have the same magnitude but opposite direction for parallel surfaces.

Let $\boldsymbol{C}^{\circ}$ and $\boldsymbol{A}^{\circ}$ be the $4^{\text {th }}$ order elasticity tensors of a reference linear medium. These are defined as the Voigt average of the stiffness tensors, $\boldsymbol{C}^{i}$ and $\boldsymbol{A}^{i}$, of all grains $i$ in the polycrystal i.e. $\boldsymbol{C}^{\circ}=\left\langle\boldsymbol{C}^{i}\right\rangle$ and $\boldsymbol{A}^{\circ}=\left\langle\boldsymbol{A}^{i}\right\rangle$. Although not proven to be the optimum choice for the linear reference medium, Voigt averages are usually adopted for their simplicity and convergent behavior $[49,50]$. Recalling that the total strain and curvatures are defined as gradients of total displacements, the Cauchy and deviatoric couple stresses 
at a material point $\overrightarrow{\boldsymbol{x}}$ can be written as,

$$
\begin{aligned}
\sigma_{i j}^{s}(\overrightarrow{\boldsymbol{x}}) & =\sigma_{i j}^{s}(\overrightarrow{\boldsymbol{x}})+C_{i j k l}^{\circ} u_{k, l}(\overrightarrow{\boldsymbol{x}})-C_{i j k l}^{\circ} u_{k, l}(\overrightarrow{\boldsymbol{x}}) \\
M_{i j}^{D}(\overrightarrow{\boldsymbol{x}}) & =M_{i j}^{D}(\overrightarrow{\boldsymbol{x}})+\frac{1}{2} A_{i j k l}^{\circ} e_{k m n} u_{n, m l}(\overrightarrow{\boldsymbol{x}})-\frac{1}{2} A_{i j k l}^{\circ} e_{k m n} u_{n, m l}(\overrightarrow{\boldsymbol{x}})
\end{aligned}
$$

Defining the Cauchy stress polarization $\left(\tau_{i j}\right)$ and couple stress polarization $\left(\mu_{i j}\right)$ tensors as,

$$
\begin{aligned}
\tau_{i j}(\overrightarrow{\boldsymbol{x}}) & =\sigma_{i j}^{s}(\overrightarrow{\boldsymbol{x}})-C_{i j k l}^{\circ} u_{k, l}(\overrightarrow{\boldsymbol{x}}) \\
\mu_{i j}(\overrightarrow{\boldsymbol{x}}) & =M_{i j}^{D}(\overrightarrow{\boldsymbol{x}})-\frac{1}{2} A_{i j k l}^{\circ} e_{k m n} u_{n, m l}(\overrightarrow{\boldsymbol{x}})
\end{aligned}
$$

the local Cauchy and couple stresses can be redefined as,

$$
\begin{aligned}
\sigma_{i j}^{s}(\overrightarrow{\boldsymbol{x}}) & =\tau_{i j}(\overrightarrow{\boldsymbol{x}})+C_{i j k l}^{\circ} u_{k, l}(\overrightarrow{\boldsymbol{x}}) \\
M_{i j}^{D}(\overrightarrow{\boldsymbol{x}}) & =\mu_{i j}(\overrightarrow{\boldsymbol{x}})+\frac{1}{2} A_{i j k l}^{\circ} e_{k m n} u_{n, m l}(\overrightarrow{\boldsymbol{x}})
\end{aligned}
$$

The polarization tensors indicate the fluctuation, from the volume average, of the local stress fields. Substituting (26) and (27) in the equilibrium equation (14) gives

$$
\begin{aligned}
\sigma_{i j, j}^{s}(\overrightarrow{\boldsymbol{x}})+\frac{1}{2} e_{i j k} M_{k l, l j}^{D}(\overrightarrow{\boldsymbol{x}}) & =C_{i j k l}^{\circ} u_{k, l j}(\overrightarrow{\boldsymbol{x}})+\frac{1}{4} e_{i j k} A_{k l m n}^{\circ} e_{m o p} u_{p, o n l j}(\overrightarrow{\boldsymbol{x}}) \\
& +\tau_{i j, j}(\overrightarrow{\boldsymbol{x}})+\frac{1}{2} e_{i j k} \mu_{k l, l j}(\overrightarrow{\boldsymbol{x}})=0
\end{aligned}
$$

Let a fictitious body force $\overrightarrow{\boldsymbol{f}}$ be defined as,

$$
f_{i}(\overrightarrow{\boldsymbol{x}})=\tau_{i j, j}(\overrightarrow{\boldsymbol{x}})+\frac{1}{2} e_{i j k} \mu_{k l, l j}(\overrightarrow{\boldsymbol{x}})
$$

then the displacement can be defined as a convolution of the Green's function $\boldsymbol{G}$ and the fictitious body force as,

$$
u_{k}(\overrightarrow{\boldsymbol{x}})=\int G_{k i}\left(\overrightarrow{\boldsymbol{x}}-\overrightarrow{\boldsymbol{x}}^{\prime}\right) f_{i}\left(\overrightarrow{\boldsymbol{x}}^{\prime}\right) d \overrightarrow{\boldsymbol{x}}^{\prime}
$$

Defining $F_{i j l n o p}^{\circ}=\frac{1}{4} e_{i j k} A_{k l m n}^{\circ} e_{m o p}$ and substituting the displacement by the Green's function in the equilibrium equation (28) gives,

$$
C_{i j k l}^{\circ} G_{k q, l j}\left(\overrightarrow{\boldsymbol{x}}-\overrightarrow{\boldsymbol{x}}^{\prime}\right)+F_{i j l n o p}^{\circ} G_{p q, o n l j}\left(\overrightarrow{\boldsymbol{x}}-\overrightarrow{\boldsymbol{x}}^{\prime}\right)+\delta_{i q} \boldsymbol{\delta}\left(\overrightarrow{\boldsymbol{x}}-\overrightarrow{\boldsymbol{x}}^{\prime}\right)=0
$$


where $\boldsymbol{\delta}$ is the Dirac delta function and $\delta_{i q}$ is the Kronecker delta. This equation represents a couple stress based extension of the periodic Lippmann Schwinger equation where the Green's tensor $G_{i j}\left(\overrightarrow{\boldsymbol{x}}-\overrightarrow{\boldsymbol{x}}^{\prime}\right)$ represents the displacement $u_{i}$ at point $\overrightarrow{\boldsymbol{x}}$ due to a unit force $f_{j}$ at point $\overrightarrow{\boldsymbol{x}}^{\prime}$ arising from the local polarization of Cauchy and couple stress fields in a homogeneous medium.

Replacing the index $p$ by $k$, performing the Fourier transform and rearranging the terms gives the following expression for the Green's tensor,

$$
\hat{G}_{k i}(\overrightarrow{\boldsymbol{k}})=\left(k_{l} k_{j} C_{i j k l}^{\circ}-k_{o} k_{n} k_{l} k_{j} F_{i j l n o k}^{\circ}\right)^{-1}
$$

where the superscript $\wedge$ represents the Fourier transform of a spatial function and $\overrightarrow{\boldsymbol{k}}$ represents a point in the Fourier space whose magnitude is equal to the angular frequency. Taking the Fourier transform of the fictitious body force in equation (29) gives,

$$
\hat{f}_{i}(\overrightarrow{\boldsymbol{k}})=i k_{j} \hat{\tau}_{i j}(\overrightarrow{\boldsymbol{k}})-\frac{1}{2} e_{i j k} k_{l} k_{j} \hat{\mu}_{k l}(\overrightarrow{\boldsymbol{k}})
$$

Finally, the compatible local total strain and curvature are

$$
\begin{aligned}
\epsilon_{i j}(\overrightarrow{\boldsymbol{x}}) & =E_{i j}+\frac{1}{2} F T^{-1}\left(i k_{j}\left(\hat{G}_{i k}(\overrightarrow{\boldsymbol{k}})+\hat{G}_{k i}(\overrightarrow{\boldsymbol{k}})\right) \hat{f}_{k}(\overrightarrow{\boldsymbol{k}})\right) \\
\kappa_{i j}(\overrightarrow{\boldsymbol{x}}) & =K_{i j}-\frac{1}{2} e_{i k l} F T^{-1}\left(k_{j} k_{k} \hat{G}_{l m}(\overrightarrow{\boldsymbol{k}}) \hat{f}_{m}(\overrightarrow{\boldsymbol{k}})\right)
\end{aligned}
$$

where $F T^{-1}$ is the inverse Fourier transform operator. The terms associated with this operator are the fluctuating terms from equation (21).

\subsection{Iterative procedure}

An Euler implicit time discretization scheme is employed to solve the local problem. The Cauchy and deviatoric couple stresses at a material point $\overrightarrow{\boldsymbol{x}}$ at time $t+\Delta t$ are expressed as:

$$
\begin{gathered}
\boldsymbol{\sigma}^{s(t+\Delta t)}(\overrightarrow{\boldsymbol{x}})=\boldsymbol{C}(\overrightarrow{\boldsymbol{x}}):\left(\boldsymbol{\epsilon}^{(t+\Delta t)}(\overrightarrow{\boldsymbol{x}})-\boldsymbol{\epsilon}^{p(t)}(\overrightarrow{\boldsymbol{x}})-\dot{\boldsymbol{\epsilon}}^{p(t+\Delta t)}(\overrightarrow{\boldsymbol{x}}) \Delta t\right) \\
\boldsymbol{M}^{D(t+\Delta t)}(\overrightarrow{\boldsymbol{x}})=\boldsymbol{A}(\overrightarrow{\boldsymbol{x}}):\left(\boldsymbol{\kappa}^{(t+\Delta t)}(\overrightarrow{\boldsymbol{x}})-\boldsymbol{\kappa}^{p(t)}(\overrightarrow{\boldsymbol{x}})-\dot{\boldsymbol{\kappa}}^{p(t+\Delta t)}(\overrightarrow{\boldsymbol{x}}) \Delta t\right)
\end{gathered}
$$

At the zeroeth time step, initialization of the curvatures (elastic, plastic and total) can be done using two techniques: (a) let the estimate of initial curvatures be equal to the initial elastic curvatures and neglect the plastic 
curvature history of the material. Therefore, total curvatures are equal to the elastic curvatures. (b) Prescribe these initial curvatures as negative plastic curvatures or 'eigencurvatures' (in the sense of eigenstrains). The total curvatures are initialized to zero and the elastic curvatures are the outcome of the numerical procedure. The latter approach is chosen in this work for the following reasons: (i) at the first iteration of the first time step, the couple stresses generated using the former approach do not respect equilibrium condition, (ii) the eigencurvature approach computes the elastic curvatures that respect the kinematic conditions associated with the evolving GB structure which is not the case with the former technique where initial elastic curvatures do not evolve. The eigencurvature approach allows defining initial polar densities in a manner that is phenomenologically consistent; it captures the GB defect content due to prior deformation. All the stresses and strains are initialized to zero.

After initialization, the solution algorithm follows steps similar to the EVP FFT model of Lebensohn et al. [50]. The first step involves computing $\boldsymbol{\tau}$ and $\boldsymbol{\mu}$. If $\boldsymbol{e}^{(i)}, \boldsymbol{\varkappa}^{(i)}, \boldsymbol{\lambda}^{(i)}$ and $\boldsymbol{\phi}^{(i)}$ are the guesses for strain, curvature, Cauchy stress, and couple stress, respectively, at the iteration $i$ then,

$$
\begin{aligned}
\tau_{i j}^{(i)}(\overrightarrow{\boldsymbol{x}}) & =\lambda_{i j}^{(i)}(\overrightarrow{\boldsymbol{x}})-C_{i j k l}^{o} e_{k l}^{(i)}(\overrightarrow{\boldsymbol{x}}) \\
\mu_{i j}^{(i)}(\overrightarrow{\boldsymbol{x}}) & =\phi_{i j}^{(i)}(\overrightarrow{\boldsymbol{x}})-A_{i j k l}^{o} \varkappa_{k l}^{(i)}(\overrightarrow{\boldsymbol{x}})
\end{aligned}
$$

Green's tensor and the $i+1$ guess for the fictive body force in Fourier space are then computed using equations (32) and (33). These equations along with the polarization tensors in equations (38) and (39) give the $(i+1)$ guesses for compatible total strain and curvature:

$$
\begin{aligned}
& e_{i j}^{(i+1)}(\mathbf{x})=E_{i j}+\frac{1}{2} F T^{-1}\left(i k_{j}\left(\hat{G}_{i k}(\overrightarrow{\boldsymbol{k}})+\hat{G}_{k i}(\overrightarrow{\boldsymbol{k}})\right) \hat{f}_{k}^{(i)}(\overrightarrow{\boldsymbol{k}})\right) \\
& \varkappa_{i j}^{(i+1)}(\mathbf{x})=K_{i j}-\frac{1}{2} e_{i k l} F T^{-1}\left(k_{j} k_{k} \hat{G}_{l m}(\overrightarrow{\boldsymbol{k}}) \hat{f}_{m}^{(i)}(\overrightarrow{\boldsymbol{k}})\right)
\end{aligned}
$$

The stresses can then be computed using equations (36) and (37). However, such a direct computation does not ensure that the constitutively obtained stresses fulfill the equilibrium conditions, thus taking a lot of iterations to converge.

In order to achieve a faster convergence, an augmented Lagrangian scheme proposed in the work of Michel et al. $[65,66]$ is employed. The original scheme based on classical continuum adjusts two strain and Cauchy stress 
fields such that one strain field is compatible $(\boldsymbol{e})$ (similar to the one in equation (34) but in classical elasticity) and one Cauchy stress field $(\boldsymbol{\lambda})$ satisfies equilibrium (only the components related to Cauchy stresses in equation $(28))$. The other strain $(\boldsymbol{\epsilon})$ and Cauchy stress $(\boldsymbol{\sigma})$ are constitutively related. The iterative procedure, a non-linear Newton-Raphson scheme, is designed such that the two strains and two stresses converge to each other. Following convergence, the compatible strain field is constitutively related to an equilibrated stress field. In the following, an extension of this augmented Lagrangian technique is employed where along with the strains and stresses, constitutively related curvature $(\boldsymbol{\kappa})$ and couple stress $(\boldsymbol{M})$ are adjusted to compatible curvatures $(\varkappa)$ and equilibrated couple stresses $(\phi)$, respectively.

This procedure involves transforming the Cauchy and couple stresses, strain, curvature, and stiffness tensors $\boldsymbol{A}$ and $\boldsymbol{C}$ into a new basis obtained using the Kelvin decomposition. For Cauchy stresses, strains and stiffness tensor $\boldsymbol{C}$, a 6 -dimensional basis $\left(\mathbb{B}^{6}\right)$ is typically used due to the symmetry of the former two tensors $[49,67]$. For the case of couple stresses, curvatures and stiffness tensor $\boldsymbol{A}$, which have 9 independent components ( 8 if deviatoric couple stresses and curvatures are considered), a 9 -dimensional basis $\left(\mathbb{B}^{9}\right)$ is used. Detailed derivation for the Kelvin decomposition to obtain $\left(\mathbb{B}^{9}\right)$ bases is shown in Appendix A.

For each material point $\overrightarrow{\boldsymbol{x}}$, the enhanced augmented Lagrangian scheme requires minimizing the residual $R_{i}$ :

$$
\begin{aligned}
& R_{i}\left(\boldsymbol{\sigma}^{s(i+1)}, \boldsymbol{M}^{D(i+1)}\right)(\overrightarrow{\boldsymbol{x}}) \\
& =\left\{\begin{array}{c}
\sigma_{k}^{s(i+1, j)}(\overrightarrow{\boldsymbol{x}})+C_{k l}^{0} \epsilon_{l}^{(i+1)}(\overrightarrow{\boldsymbol{x}})-\lambda_{k}^{(i)}(\overrightarrow{\boldsymbol{x}})-C_{k l}^{0} e_{l}^{(i+1)}(\overrightarrow{\boldsymbol{x}}) ; k, l=1-6 \\
M_{k}^{D(i+1, j)}(\overrightarrow{\boldsymbol{x}})+A_{k l}^{0} \kappa_{l}^{(i+1)}(\overrightarrow{\boldsymbol{x}})-\phi_{k}^{(i)}(\overrightarrow{\boldsymbol{x}})-A_{k l}^{0} \varkappa_{l}^{(i+1)}(\overrightarrow{\boldsymbol{x}}) ; k, l=7-15
\end{array}\right\}
\end{aligned}
$$

The eigencurvatures are introduced at the first iteration of the first time step as plastic curvatures in equation (42) which are incorporated in $\kappa_{l}$ along with the elastic curvatures.

The 15 non-linear equations in (42) are solved using the Newton-Raphson iterative procedure as follows:

$$
\begin{aligned}
& \sigma_{k}^{s(i+1, j+1)}=\sigma_{k}^{s(i+1, j)}-\left[\left.\frac{\partial R_{k}}{\partial \sigma_{l}}\right|_{\boldsymbol{\sigma}^{s(i+1, j)}, \boldsymbol{M}^{D(i+1, j)}}\right]^{-1} R_{l}\left(\boldsymbol{\sigma}^{s(i+1, j)}, \boldsymbol{M}^{D(i+1, j)}\right) \\
& M_{k}^{D(i+1, j+1)}=M_{k}^{D(i+1, j)}-\left[\left.\frac{\partial R_{k}}{\partial M_{l}^{D}}\right|_{\boldsymbol{\sigma}^{s(i+1, j)}, \boldsymbol{M}^{D(i+1, j)}}\right]^{-1} R_{l}\left(\boldsymbol{\sigma}^{s(i+1, j)}, \boldsymbol{M}^{D(i+1, j)}\right)(
\end{aligned}
$$


where the residual computed at $(j+1)$ iteration of the Newton-Raphson loop is used to obtain $(j+1)$ guess for the stresses. The inverse term is the $15 \times 15$ Jacobian matrix obtained by taking the partial derivative of the residual with respect to Cauchy and couple stresses gives the following expression for the Jacobian:

$$
\begin{aligned}
& \left.\frac{\partial R_{k}}{\partial \sigma_{l}^{s}}\right|_{\boldsymbol{\sigma}^{s(i+1, j)}, \boldsymbol{M}^{D(i+1, j)}}=\delta_{k l}+C_{k m}^{0} C_{m l}^{-1}+C_{k m}^{0} \frac{\partial \dot{\epsilon}_{m}^{p}}{\partial \sigma_{l}^{s}} \Delta t ; k, l, m=1-6 \\
& \left.\frac{\partial R_{k}}{\partial \sigma_{l}^{s}}\right|_{\boldsymbol{\sigma}^{s(i+1, j)}, M^{D(i+1, j)}}=A_{k m}^{0} \frac{\partial \dot{\kappa}_{m}^{p}}{\partial \sigma_{l}^{s}} \frac{\Delta t}{\sqrt{2}} \quad ; k, m=7-15, l=1-6 \\
& \left.\frac{\partial R_{k}}{\partial M_{l}^{D}}\right|_{\boldsymbol{\sigma}^{s(i+1, j)}, M^{D(i+1, j)}}=0 \quad ; k=1-6, l=7-15 \\
& \left.\frac{\partial R_{k}}{\partial M_{l}^{D}}\right|_{\boldsymbol{\sigma}^{s(i+1, j)}, \boldsymbol{M}^{D(i+1, j)}}=\delta_{k l}+A_{k m}^{0} A_{m l}^{-1}+A_{k m}^{0} \frac{\partial \dot{\kappa}_{m}^{p}}{\partial M_{l}^{D}} \Delta t ; k, l, m=7-15
\end{aligned}
$$

where the terms associated with $\Delta t$, i.e. $\frac{\partial \dot{\epsilon}_{m}^{p}}{\partial \sigma_{l}^{s}}, \frac{\partial \dot{\kappa}_{m}^{p}}{\partial \sigma_{l}^{s}}$ and $\frac{\partial \dot{\kappa}_{m}^{p}}{\partial M_{l}^{s}}$, are the tangent compliance in classical viscoplasticity, curvature based compliance in classical continuum and couple stress based compliance in higher order continuum, respectively. Note that in the present work we are only concerned in studying the nucleation of disclinations and not their evolution, the higher order continuum compliance tensor $\frac{\partial \dot{\kappa}_{m}^{p}}{\partial M_{l}^{s}}$ is taken equal zero. Also, the contribution of $\frac{\partial \dot{\kappa}_{m}^{p}}{\partial \sigma_{l}^{s}}$ is neglected. Apart from the complexity of the expression, its contraction with the couple stress component of the residual in equation 42 results in a negligible contribution to the Cauchy stress. This assumption also complements the Cauchy stress elastic constitutive relationship (15) which is assumed to be independent of the elastic curvatures.

The critical resolved shear stress (CRSS) $\tau_{0}$, which is a function of the stress dependent plastic strain, is taken as a function of stress $\tau_{0}^{s}\left(\boldsymbol{\epsilon}^{p}(\boldsymbol{\sigma})\right)=$ $\tau_{0}^{s}(\boldsymbol{\sigma})$ such that:

$$
\frac{\partial \dot{\epsilon}_{i}^{p}}{\partial \sigma_{j}^{s}} \approx n \dot{\gamma}_{0} \sum_{\alpha=1}^{N} \frac{m_{i}^{\alpha} m_{j}^{\alpha}}{\tau_{0}^{s}\left(\boldsymbol{\sigma}^{s(i+1, j)}\right)}\left(\frac{\left|\boldsymbol{m}^{\boldsymbol{\alpha}} \cdot \boldsymbol{\sigma}^{\boldsymbol{s}}\right|}{\tau_{0}^{s}\left(\boldsymbol{\sigma}^{s(i+1, j)}\right)}\right)^{n-1}
$$

where $\boldsymbol{m}^{\alpha}$ is the symmetric Schmid tensor $\mathbf{P}^{\alpha}$ in basis $B$. Note here that the multiplicant associated with the partial derivative of CRSS is omitted. Indeed this results in an approximate form of the Jacobian, but has a negligible effect on the convergence rate [50]. Once convergence is achieved, the 
new guess for auxiliary Cauchy and couple stress fields is given by,

$$
\begin{gathered}
\boldsymbol{\lambda}^{(i+1)}(\overrightarrow{\boldsymbol{x}})=\boldsymbol{\lambda}^{(i)}(\overrightarrow{\boldsymbol{x}})+\boldsymbol{C}^{0}:\left(\boldsymbol{e}^{(i+1)}(\overrightarrow{\boldsymbol{x}})-\boldsymbol{\epsilon}^{(i+1)}(\overrightarrow{\boldsymbol{x}})\right) \\
\boldsymbol{\phi}^{(i+1)}(\overrightarrow{\boldsymbol{x}})=\boldsymbol{\phi}^{(i)}(\overrightarrow{\boldsymbol{x}})+\boldsymbol{A}^{0}:\left(\varkappa^{(i+1)}(\overrightarrow{\boldsymbol{x}})-\boldsymbol{\kappa}^{(i+1)}(\overrightarrow{\boldsymbol{x}})\right)
\end{gathered}
$$

The iterations are repeated until normalized average differences between $\boldsymbol{\sigma}(\overrightarrow{\boldsymbol{x}})$ and $\boldsymbol{\lambda}(\overrightarrow{\boldsymbol{x}}), \boldsymbol{M}(\overrightarrow{\boldsymbol{x}})$ and $\boldsymbol{\phi}(\overrightarrow{\boldsymbol{x}}), \boldsymbol{\epsilon}(\overrightarrow{\boldsymbol{x}})$ and $\boldsymbol{e}(\overrightarrow{\boldsymbol{x}})$, and $\boldsymbol{\kappa}(\overrightarrow{\boldsymbol{x}})$ and $\boldsymbol{\varkappa}(\overrightarrow{\boldsymbol{x}})$, are smaller than a threshold.

Higher order boundary conditions in the form of macroscopic total strain and curvature rate are imposed on the periodic microstructure such that at time step $t+1$ the macroscopic strain and curvature are given as,

$$
\begin{aligned}
& E_{i j}^{(t+1)}=E_{i j}^{(t)}+\dot{E}_{i j} \Delta t \\
& K_{i j}^{(t+1)}=K_{i j}^{(t)}+\dot{K}_{i j} \Delta t
\end{aligned}
$$

In addition to these, macroscopic Cauchy $\boldsymbol{\Sigma}$ and couple $\boldsymbol{\Phi}$ stresses can also be imposed. These boundary conditions allow for the simulation of pure tension/compression, pure shear, pure bending, pure torsion, creep (in tension, bending or torsion) and complex loading scenarios. Prescribing the macroscopic stresses requires modifying the macroscopic strain and curvature at each iteration $i+1$ by the following:

$$
\begin{aligned}
& E_{i j}^{(t+1, i+1)}=E_{i j}^{(t+1, i)}+C_{i j k l}^{o-1} \alpha^{k l}\left(\Sigma_{k l}-\left\langle\lambda_{k l}^{(i+1)}(\mathbf{x})\right\rangle\right) \dot{E}_{i j} \Delta t \\
& K_{i j}^{(t+1, i+1)}=K_{i j}^{(t+1, i)}+A_{i j k l}^{o-1} \beta^{k l}\left(\Phi_{k l}-\left\langle\phi_{k l}^{(i+1)}(\mathbf{x})\right\rangle\right) \dot{K}_{i j} \Delta t
\end{aligned}
$$

where $\alpha^{k l}=1$ and $\beta^{k l}=1$ if components $\Sigma_{k l}$ and $\Phi_{k l}$ are imposed, respectively, and zero otherwise. A flow chart/block diagram for the CSEVP FFT model is shown in Appendix C.

\subsection{Gibbs phenomenon and its discrete Fourier transform based numerical correction}

In the FFT approach, the continuous Fourier transform of a piecewise continuous function (say $\mho$ ) is approximated as a discrete sum of a Fourier series defined using sine and cosine functions. The domain, in which the piecewise continuous functions are defined, is divided into a grid of equidis-

tant points, known as Fourier points, at which the governing equations are solved. 
If a local discontinuity exists in an otherwise piecewise continuous function $\mho$, using the FFT approach results in spurious oscillations in $\hat{\mho}$ the vicinity of this discontinuity. The formation of these spurious oscillations is known as the Gibbs phenomenon. In the context of present work, this phenomenon would be incurred in computing the strains (Cauchy stress) and curvatures (couple stress) using equations (34) and (35), respectively, due to discontinuous initial curvatures and inhomogeneous elastic constants $\boldsymbol{C}$ at the interface.

The Gibbs phenomenon is demonstrated using two bicrystalline microstructures having crystals forming an asymmetric mixed tilt and twist high angle GB (i) without initial curvatures and (ii) with initial curvatures. The bicrystal and associated Euler angles are shown in figures 1(a) and (b). In both cases the inter Fourier point distance in all directions is $0.7252 \mathrm{~nm}$, the shortest distance used in this work. This gives large magnitudes of initial curvatures. An exaggerated estimate of initial curvatures is used to characterize the bi-crystal; the first and second neighbor Fourier points to the interface are assigned three times the actual value of estimated curvatures. A detailed explanation on assigning the initial curvatures is given in section 5. Both bicrystals are loaded in uniaxial tension along the direction perpendicular to GB plane up to $1.2 \%$ strain while disallowing plasticity. Gibbs phenomenon due to inhomogeneity in stiffness matrix $\boldsymbol{C}$ is found to be negligible compared to the one introduced by initial curvatures. Focusing on the effect of latter, the evolution of $\kappa_{32}^{e}$ and $\sigma_{12}$ in the direction perpendicular to GB interfaces are shown in figure 1(c) and (d). The large spurious fluctuations confirm the occurrence of the Gibbs phenomenon.

A correction to the Gibbs phenomenon is possible by replacing continuous Fourier transforms (CFTs) of partial derivatives of field quantities with their discrete Fourier transforms (DFTs). The DFTs are obtained by Fourier transforming centered difference approximation of the partial derivatives [6871]. In the present work, the DFTs of first and second order partial derivatives are of interest; the procedure to obtain the 3-dimensional derivatives is detailed in Appendix B. Using equation (33) for the fictitious body force and defining a modified Green's tensor $\hat{\Gamma}_{i j k l}=k_{j} k_{l} \hat{G}_{i k}(\overrightarrow{\boldsymbol{k}})$, equations (40) and (41) can be rewritten as: 


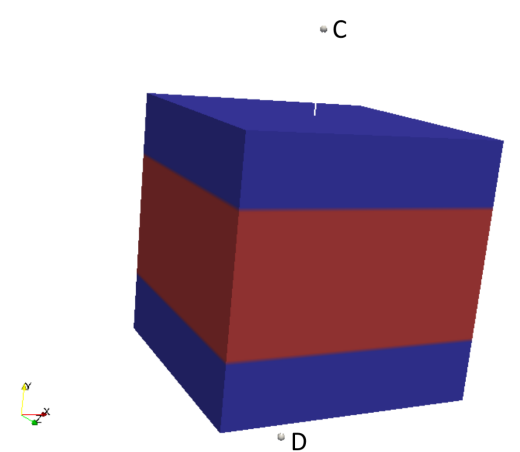

(a) Bicrystal

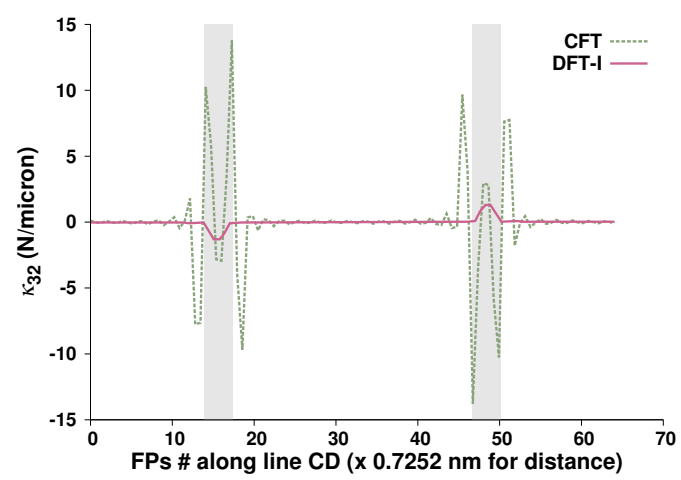

(c) $\kappa_{32}^{e}(\mathrm{~N} /$ micron $)$

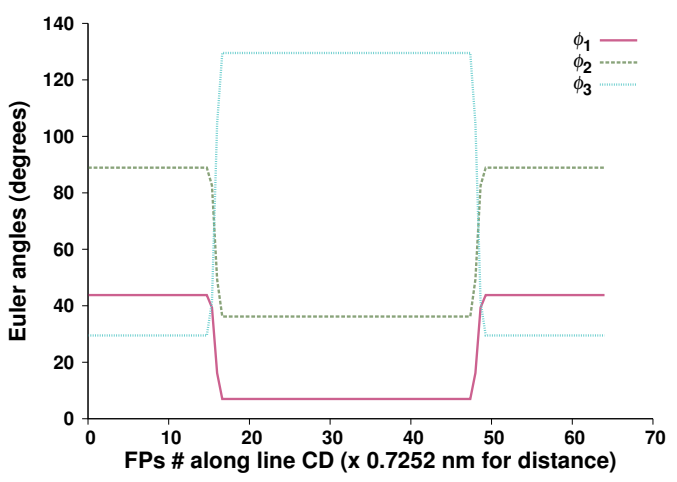

(b) Euler angles

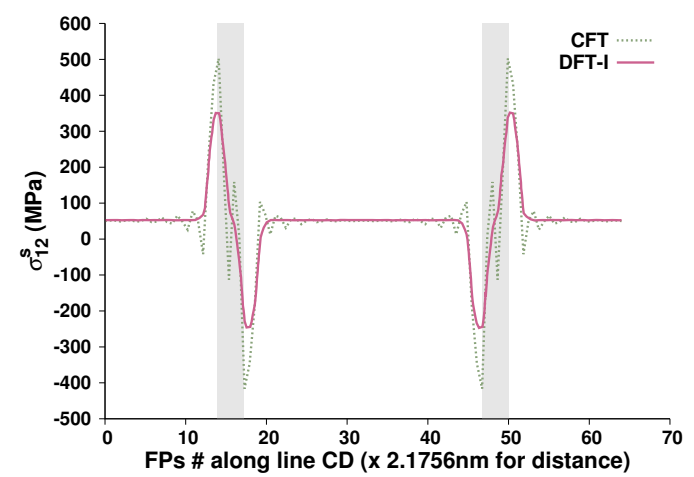

(d) $\sigma_{12}(\mathrm{MPa})$

Figure 1: (a) Microstructure of bicrystal with planar (b) mixed tilt and twist high angle GB, plotted along the GB normal direction. (c) Elastic curvature component $\kappa_{32}^{e}$ $\left(\mathrm{N} /\right.$ micron) and (d) Cauchy stress $\sigma_{12}(\mathrm{MPa})$ plotted along line CD for bicrystal-b configuration, respectively, at $1.2 \%$ applied strain along GB normal using CFT and DFT-I procedures. The thickness of gray lines is representative of the GB interface thickness i.e. number of Fourier points which are assigned the initial curvature.

$$
\begin{aligned}
& e_{i j}(\mathbf{x})=E_{i j}-\frac{1}{2} F T^{-1}\left(\left(\hat{\Gamma}_{i j k l}(\overrightarrow{\boldsymbol{k}})+\hat{\Gamma}_{j i k l}(\overrightarrow{\boldsymbol{k}})\right)\left(\hat{\tau}_{k l}(\overrightarrow{\boldsymbol{k}})-\frac{1}{2} e_{k l m}\left(i k_{n}\right) \hat{\mu}_{m n}(\overrightarrow{\boldsymbol{k}})\right)\right) \\
& \varkappa_{i j}(\mathbf{x})=K_{i j}+\frac{1}{2} e_{i k l} F T^{-1}\left(\hat{\Gamma}_{l j m k}(\overrightarrow{\boldsymbol{k}})\left(\left(i k_{n}\right) \hat{\tau}_{m n}(\overrightarrow{\boldsymbol{k}})+\frac{1}{2} e_{m n p}\left(k_{n} k_{q}\right) \hat{\mu}_{p q}(\overrightarrow{\boldsymbol{k}})\right)\right)
\end{aligned}
$$

In equations (53) and (54), continuous frequencies obtained from Fourier 
transforming first and second order partial derivaties are replaced by their DFT couterparts defined by equations (B.5) and (B.6). The modified Green's tensor, however, is computed using the CFT procedure. This procedure, henceforth named as DFT-I, gave the largest reductions in Gibbs phenomenon as is shown in figure 1(c) and (d). Using the DFT procedure for the modified Green's tensor (called DFT-2) resulted in additional inaccuracies and therefore not used. Furthermore, stresses computed using DFT-I better respect local equilibrium than the ones predicted using the CFT. This can be appreciated from the comparison of the norm of higher order equilibrium vector $\sigma_{i j, j}^{s}+\frac{1}{2} e_{i j k} M_{k l, l j}^{D}$, shown in figure 2, predicted by the CFT and DFT-I techniques.

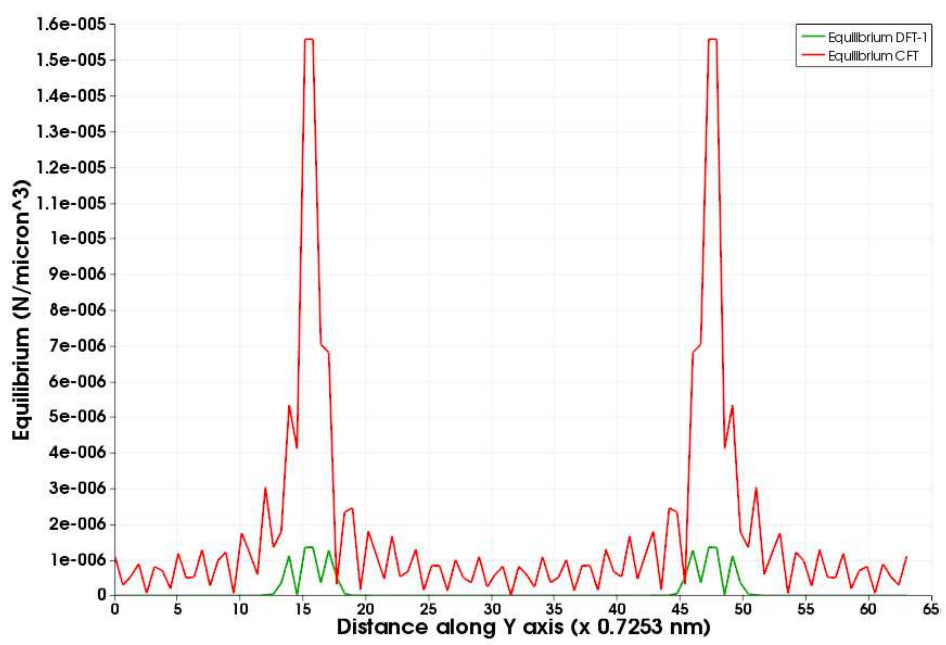

Figure 2: Comparison of the equilibrium vector magnitude computed from CFT and DFT-1 approaches at 1.2\% tensile strain along the line shown in figure 1(a) for bicrystal-a configuration.

\section{Simulation setup}

\subsection{Microstructure}

The microstructure is a periodic Voronoi cube with 100 randomly oriented grains. It is discretized into a Fourier grid of $64 \times 64 \times 64$ points; on average each grain has 2621 Fourier points associated to it. Figure 3 shows the microstructure with grains colored according to their Euler angles. Kinematic 
quantities and stress fields are studied along the line shown in figure 3(b). For the sake of simplicity, the line is oriented along the $y$ axis, in general though it is randomly chosen. The numbers represent intersection of the line with interfaces $(1,2,3,5,6,9)$, grains protruding from the normal direction $(4,7,8)$ and a triple junction $(10)$.
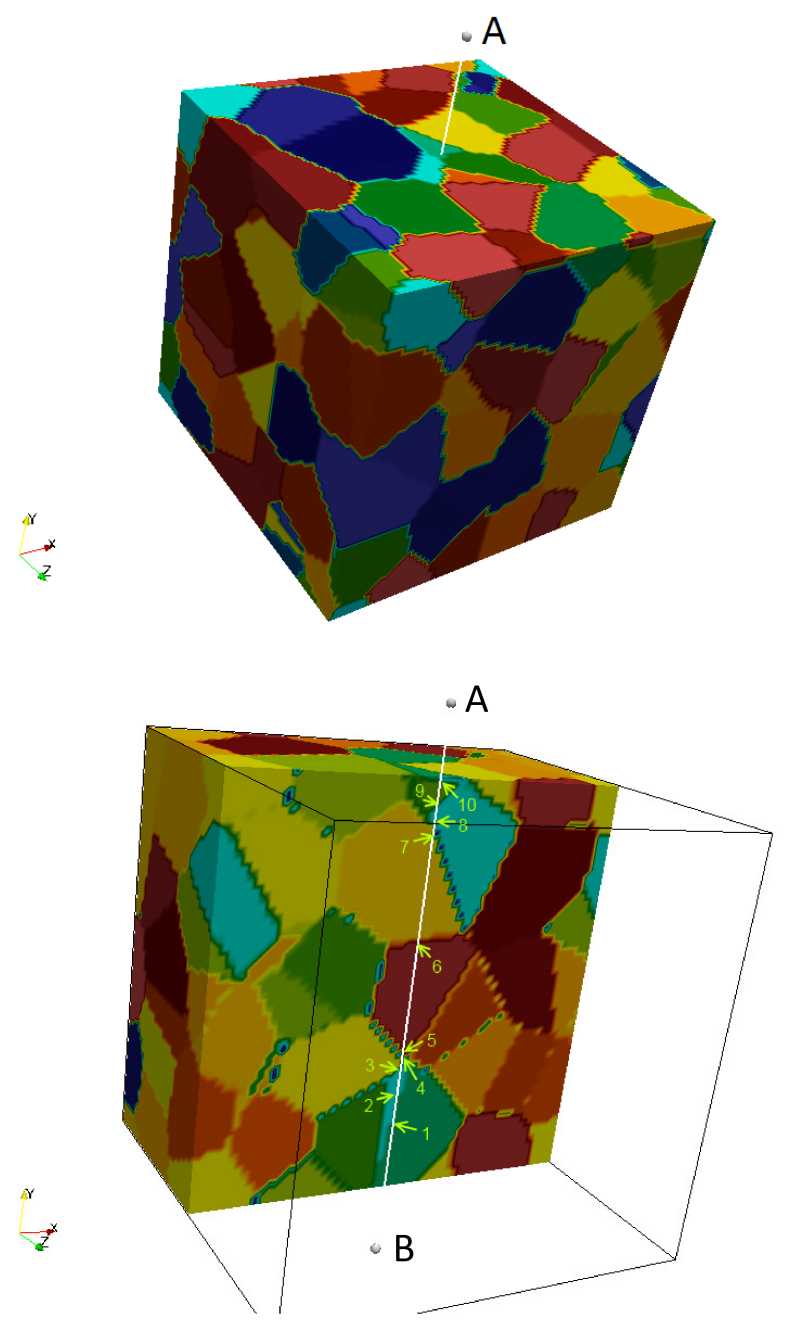

Figure 3: Simulated microstructure with 100 randomly oriented grains and $64 \times 64 \times$ 64 Fourier points. The colors represent different grains. The stress and kinematic fields are plotted along the line parallel to the $y$ direction. Numbers indicate the points of intersection of the line with an interface. 
Initial curvatures are computed using equation (20). The rigid body rotation field is obtained from the Euler angles of grains forming the boundary. The distance $\Delta x_{j}$ is assigned the inter-Fourier point distance in each direction. Three cases for GB width are considered: (a) the first case corresponds to a classical continuum approach with no initial curvatures. The GB interface is assumed to be infinitesimally thin along the bisector of two Fourier points belonging to different grains. This case is identified as $t_{0}$ as shown in figure 4. (b) In the second case, Fourier points that are immediate neighbors on either side of the GB are attributed an initial curvature $\kappa_{i j}^{i n i}$ according to equation (20). The interface has a finite thickness $\delta$ with a maximum value of $t_{1}$ along any sample axis as shown in figure 4 . (c) In the third case, second neighbor Fourier points are considered to be part of GB interface whose largest thickness is equal to $3 \delta$. This is identified as the $t_{2}$ configuration in figure 4 . The corresponding initial curvatures attributed to the GB are $\kappa_{i j}^{i n i} / 3$ in order to maintain the grain orientation across the boundary. Initial curvatures for Fourier points in the vicinity of multiple GBs have an additive contribution from all the boundaries. For the microstructure considered, the volume fraction occupied by the so-formed GBs are $0 \%$ for $t_{0}, 31 \%$ for $t_{1}$, and $55 \%$ for $t_{2}$. Finally, eigencurvatures are defined as the negative of these initial curvatures.

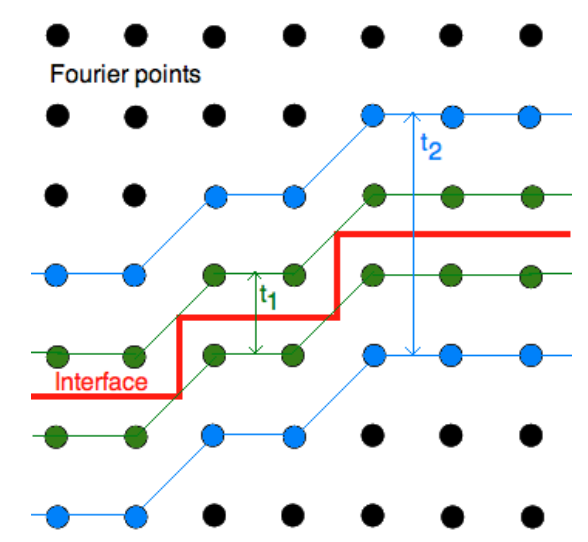

Figure 4: A 2-dimensional illustration of infinitesimally thin $t_{0}$ (in red), thickness $t_{1}=\delta$ (in green), and thickness $t_{2}=3 \delta$ (in blue), interfaces in the discretized Fourier grid consisting of evenly spaced Fourier points.

Simulations are performed for different average grain sizes viz. 10nm, 
$20 \mathrm{~nm}, 40 \mathrm{~nm}, 50 \mathrm{~nm}$ and $100 \mathrm{~nm}$. Assuming that each grain is cubic shaped and the inter Fourier point distances are same in each direction, then the latter are $0.7252 \mathrm{~nm}, 1.4504 \mathrm{~nm}$ and $2.9008 \mathrm{~nm}, 3.626 \mathrm{~nm}$ and $7.252 \mathrm{~nm}$, respectively. From equation (20), the magnitude of initial curvatures strongly depend on the values of $\Delta x_{j}$ corresponding to the inter Fourier point distance. For example, electron backscattering diffraction techniques used to compute curvatures $[72,73]$ typically have a resolution of a few microns to a few tens of microns (recent advances have better resolution on the order of hundred nanometers $[74,75])$, while the resolution of automated crystal orientation mapping using transmission electron microscopy is a few nanometers [76]. For the same microstructure, curvatures obtained from these two techniques would have very different magnitudes. Assuming that the source of the curvatures is unknown it can be justified to use the full magnitude of $\kappa_{i j}^{i n i}$ as shown in equation (20) or a fraction of it to characterize GBs. In the present work, initial microstructures are characterized with the full magnitude of $\kappa_{i j}^{i n i}$ unless mentioned otherwise.

\subsection{Material properties}

All the microstructures are attributed material properties of $\mathrm{FCC} \mathrm{Cu}$ at room temperature. The components of $\boldsymbol{C}$ in Voigt notation are: $C_{11}=170.2$ $\mathrm{GPa}, C_{12}=114.9 \mathrm{GPa}$ and $C_{44}=61.0 \mathrm{GPa}[50,77]$. For the Cauchy stress visco-plastic constitutive relationship in equation (18), the reference plastic shear rate is taken as $\dot{\gamma}_{0}=0.1 / \mathrm{sec}$ and the power exponent is $n=10$. An FCC material has $12\{111\}<110>$ slip systems available. An elasticperfectly plastic strain response is modeled. An arbitrarily chosen CRSS of $330 \mathrm{MPa}$ is used for all the simulations. Stiffness matrix $\boldsymbol{A}$ is assumed to have an isotropic form. Using Kröner's [59] estimate, in Voigt notation the diagonal values of $\boldsymbol{A}$ are all equal to $G b^{2}=3.967 \times 10^{-9} N$. Since $\boldsymbol{A}$ is a diagonal matrix with one independent eigenvalue, it is invariant to rotational transformations. Therefore, $\boldsymbol{A}^{\circ}=\left\langle\boldsymbol{A}^{i}\right\rangle=\boldsymbol{A}$. This renders the polycrystal elastically homogeneous in $\boldsymbol{A}$, thus having no contribution to couple stress polarity; this will help highlight the contribution of initial curvatures.

\subsection{Boundary conditions}

The boundary conditions are chosen to mimic a uniaxial tensile test on dog bone shaped samples. The microstructure is loaded along the $y$ direction. A macroscopic strain rate of $\dot{E}_{22}$ is imposed along with a no shear condition i.e. $\dot{E}_{12}=\dot{E}_{13}=\dot{E}_{23}=0$. Studies are performed for two strain rates, 
$\dot{E}_{22}=1 s^{-1}$ and $\dot{E}_{22}=0.001 s^{-1}$. The material is loaded to $E_{22}=2 \%$ strain in 100 steps for both these strain rates. Surfaces normal to $x$ and $z$ directions are traction free, therefore macroscopic Cauchy stress components $\Sigma_{11}=\Sigma_{33}=0$. These surfaces are allowed to bend, therefore, the macro couple stress components $\Phi_{12}=\Phi_{32}=0$. In a dog bone sample, the arms are clamped typically constrained such that the gauge area doesn't twist $\dot{K}_{22}=0$ or bend in the direction normal to $y$ such that $\dot{K}_{21}=\dot{K}_{23}=0$. Also, it is assumed that the traction free surfaces in the gauge area do not twist, therefore $\dot{K}_{11}=\dot{K}_{33}=0$. Finally, surfaces normal to directions $x$ and $z$ are assumed to not bend implying that $\dot{K}_{13}=\dot{K}_{31}=0$.

There are two possibilities for imposing the boundary conditions. The first one is to impose the eigencurvatures and allow the local stresses to plastically relax freely before imposing the load. This approach is typically followed for the cases when eigenstrains are imposed. The extent of resulting microstructure evolution is governed by the stresses generated and duration of the relaxation process. This will be different for different magnitudes and distributions of initial curvatures. In the present work the intention is to compare the loading response with the same initial grain orientations and plastic history. For this reason we propose to use the second possibility which is to skip the intermediate relaxation step and impose the eigencurvatures and loading conditions at the same instant. This does not prevent the stress relaxations to occur at the initial steps of loading, but ensures that the microstructure configuration before loading is always the same.

\section{Results}

\subsection{Effects of a higher order model: role of curvatures in accommodating plasticity}

The CSEVP DFT-I and EVP FFT models are now applied to study the local and effective mechanical response of the microstructure without initial curvatures i.e. $t_{0}$ configuration. The macroscopic Cauchy stress v/s strain response for the $10 \mathrm{~nm}, 20 \mathrm{~nm}, 30 \mathrm{~nm}, 50 \mathrm{~nm}$ and $100 \mathrm{~nm}$ microstructures are found to be very similar. Figure 5 shows the VM Cauchy stress vs. strain plots predicted by both the models for the $20 \mathrm{~nm}$ microstructure for the two strain rates.

Both the models predict a similar strain rate dependence. Interestingly, the CSEVP DFT-I model predicts a size independent response. This is because the magnitude of local couple stresses generated are not sufficiently 


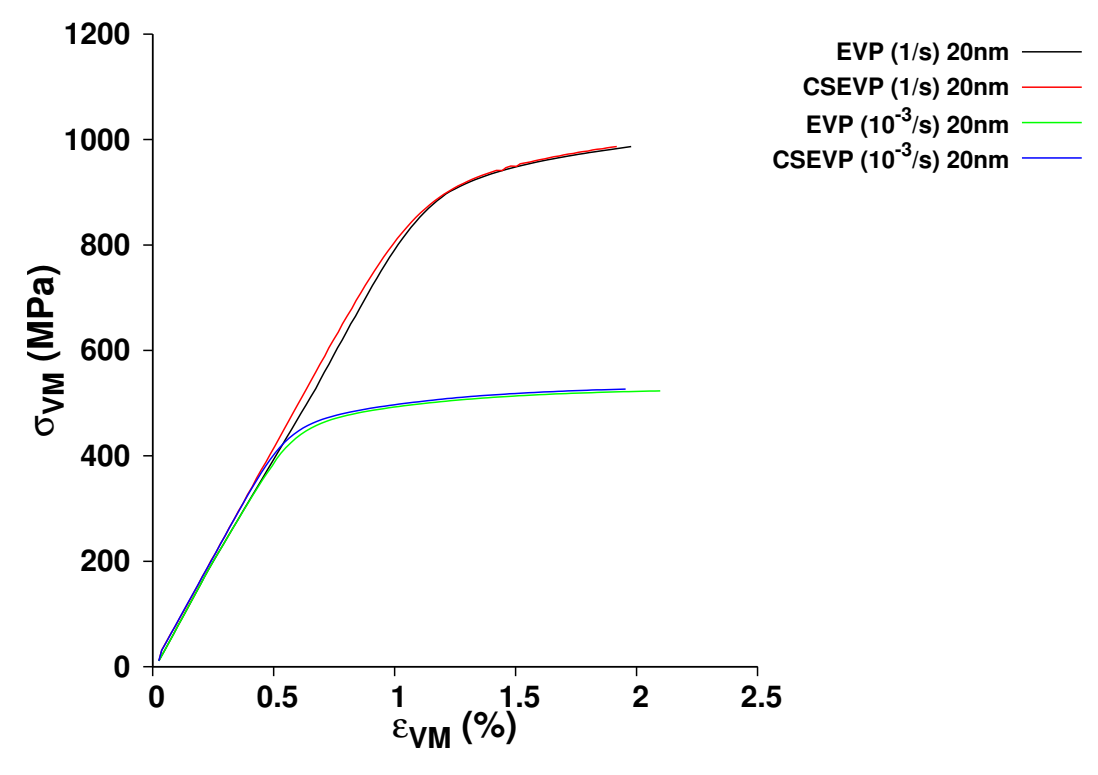

Figure 5: Comparison of VM Cauchy stress vs equivalent total strain curves predicted for $t_{0}$ microstructure by the EVP FFT and CSEVP DFT-I methods for strain rates $\dot{E}_{22}=$ $0.001 / s$ and $\dot{E}_{22}=1 / s$ upto a total strain $E_{22}=2 \%$. The response is same for all the grain sizes studied.

large to affect the Cauchy stress through the equilibrium equation, even for the case of the $10 \mathrm{~nm}$ grain size microstructure. To understand this, consider the first equilibrium equation (14) $\sigma_{11,1}+\sigma_{12,2}+\sigma_{13,3}+0.5\left(M_{31,12}+M_{32,22}+\right.$ $\left.M_{33,32}-M_{21,13}-M_{22,23}-M_{23,33}\right)$. Neglecting all the negative signs, assigning couple stress components their globally maximum value and all the Cauchy stresses their global minimum value. It is found that the contribution of couple stresses to the equilibrium equation is $10 \%$ for this hypothetical case. Note that the global maximum in couple stress magnitude occurs uniquely at one Fourier point close to an interface where the Cauchy stresses are significantly larger than their global minimum value. Therefore, although the grain size effect is captured by the CSEVP DFT-I model, it has a negligible effect on the macroscopic response.

The models differ in their prediction of the effective elastic moduli and equivalent strain at the end of loading. Focusing on the elastic response, CSEVP DFT-I always predicts a larger elastic modulus. Furthermore, for a lower applied strain, EVP FFT predicts a larger equivalent strain than 
CSEVP DFT-I. Furthermore, these differences are found to be relatively similar for all the grain sizes studied. The effect of macroscopic couple stresses and curvatures on the VM Cauchy stress vs equivalent total strain response is found to be negligible for all the different grain sizes. Then the origin of these differences should occur at the microscopic level.

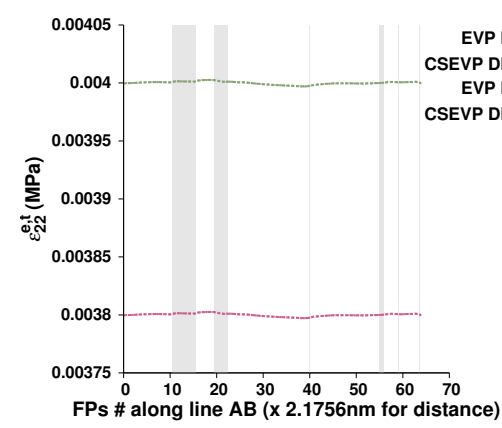

(a) $\epsilon_{22}$ and $\epsilon_{22}^{e}$ plot-over-line $\mathrm{AB}$

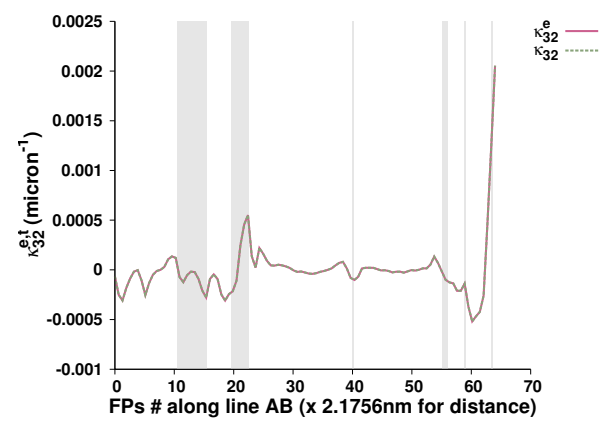

(c) $\kappa_{32}$ and $\kappa_{32}^{e}$ plot-over-line $\mathrm{AB}$

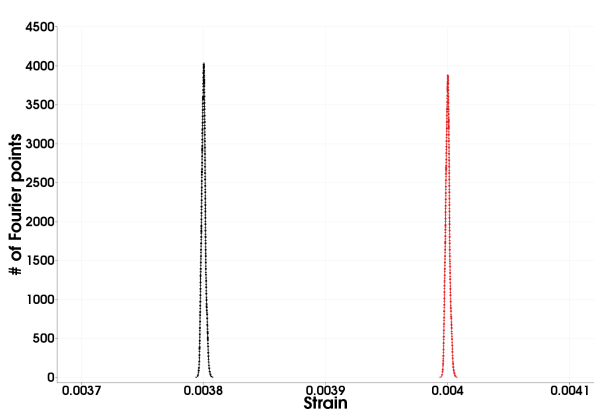

(b) $\epsilon_{22}$ and $\epsilon_{22}^{e}$ histogram

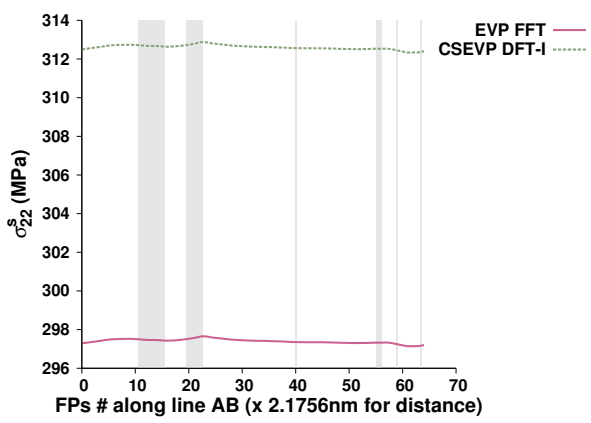

(d) $\sigma_{22}^{s}$ plot-over-line $\mathrm{AB}$

Figure 6: Comparison between EVP FFT and CSEVP DFT-I using plots over line AB for (a) $\epsilon_{22}$ and $\epsilon_{22}^{e}$, (c) $\kappa_{32}^{e},(\mathrm{e}) \sigma_{22}^{s}$. (b), (d) and (f) show their respective histograms for the $20 \mathrm{~nm}$ microstructure loaded to $0.4 \%$ strain at $E_{22}=1 / \mathrm{s}$.

Figures 6(a) and (b) show the plot over line AB (from figure 3) and the distribution of elastic and total strain along the loading direction for the microstructure loaded at $1 / s$ predicted by the CSEVP DFT-I and EVP FFT models at $0.37335 \%$ equivalent strain (an average between the equivalent strains predicted by EVP FFT -0.003726 - at $E_{22}=0.0038$ and by CSEVP DFT-I -0.003741 - at $\left.E_{22}=0.004\right)$. The histogram reveals two very sharp peaks with overlapping total and elastic strains. The average 
value of these strains corresponds to their volume averaged (macroscopic) counterparts. Similar sharp peaks are obtained for the shear stresses with their average at zero, in accordance with the BCs. These results imply that for CSEVP DFT-I to obtain the same equivalent strain at $E_{22}=0.004$ as predicted by EVP FFT at $E_{22}=0.0038$, the former model should predict a relatively lower magnitude of average strain components in the transverse directions with respect to the latter model. At applied $E_{22}=0.004$, CSEVP DFT-I predicts $E_{11}=E_{33}=-0.001612$, whereas at $E_{22}=0.0038$ EVP FFT predicts $E_{11}=E_{33}=-0.001789$. Consequently, this gives a smaller effective transverse/axial strain ratio for CSEVP DFT-I compared to EVP FFT. According to the classical theory of elasticity, for an FCC material such a difference can only occur if the Poisson's ratio is modified. However, since the elastic constants are always kept the same for both the models, therefore this change in the transverse/axial strain ratio should be a result of the higher order framework.

At the first iteration of the first time step, the applied strain results in formation of local Cauchy stress polarization field within each grain. Gradients in Cauchy stress polarization field are generated across the GB interface due to the elastic inhomogeneity of the polycrystal. On the other hand, the polarization field in couple stresses is uniformly zero everywhere in the microstructure due to the isotropic form of $\boldsymbol{A}$ (equation A.7). Then the differences in elastic moduli could arise from two possibilities (i) formulation of Green's tensor (32) used in equations (53) and (54), and (ii) Cauchy stress polarization resulting in generation of the compatible total curvatures. The first term in equation (32) corresponds to the Green's tensor in classical elasticity formulation. The second term in equation (32) is associated with the higher order elasticity tensor $\boldsymbol{F}^{\circ}$ (dependent on $\boldsymbol{A}^{\circ}$ ) and two additional frequencies which introduce a length scale dependence. As the grain size reduces, the second term in equation (32) has an increasingly significant effect on the magnitude of modified Green's tensor $\boldsymbol{\Gamma}$ in equation (53) with respect to its classical description. However, since the macroscopic elastic modulus remains the same for all grain sizes the contribution of this term to both compatible strains and curvatures should be negligible. Then a significant contribution towards accommodating the Cauchy stresses would be through the generation of compatible total curvatures from equation (54).

Figure 6(c) shows the plot over line AB for elastic and total compatible curvatures generated at $0.4 \%$ strain. As expected in the elastic regime, the total and elastic curvatures are equal. The fluctuations about the imposed 
macroscopic value of $K_{32}=0$ are much larger in the vicinity of a GB or TJ compared to grain interiors. Note that the fluctuations seemingly occurring in grain interiors are due to their proximity to a GB. During loading, local Cauchy stress polarizations generated across GB interfaces result in the generation of local compatible elastic curvatures. These local curvatures induce a transient, and fully recoverable, change in the local elastic rotations. This predominantly occurs in the vicinity of the GB and can be interpreted as a transient change in GB misorientation, thus accommodating a part of the applied load.

Relatively larger values of Cauchy stresses predicted by CSEVP DFTI (see figure 6d) for the same equivalent strain result in earlier yielding in comparison to EVP FFT. At $0.8 \%$ equivalent total strain, CSEVP DFT-I predicts the equivalent plastic strain to be $0.03646 \%$. This is $65 \%$ higher than the one obtained using EVP FFT (0.02209\%). Interestingly though, at $1.31 \%$ equivalent total strain the VM plastic strain predicted by CSEVP DFT-I (0.1962\%) is lower than the one obtained using EVP FFT (0.1974\%) for the same VM Cauchy stress of $920 \mathrm{MPa}$. This difference continues to increase till the end of loading where the VM Cauchy stress predicted by both the models is the same $(986.6 \mathrm{MPa})$ but the VM plastic strains are $0.7303 \%$ and $0.7393 \%$, respectively, for CSEVP DFT-I and EVP FFT. This is because part of the Cauchy stresses are accommodated by elastic and plastic (following on-set of plasticity) curvatures resulting in a relative reduction of the accumulated VM plastic strain predicted by CSEVP DFT-I.

Figure 7 shows the evolution of distribution of local couple stress norm $(|| \boldsymbol{M} \|)$ during deformation of the $20 \mathrm{~nm}$ microstructure loaded at a rate of $1 / s$. In the elastic regime, the distribution is skewed right i.e. the peak has a right shoulder. The intensity remains the same in the elastic regime, however, the variance increases. This is due to the elastic inhomogeneity of tensor $\boldsymbol{C}$ which locally contributes to the couple stresses through the higher order equilibrium condition. Following the onset of plasticity, mean and variance of couple stress norm continue to increase, however, now the intensities increase too. Furthermore, a distinct secondary peak with a lower intensity begins to appear. The magnitudes of couple stresses contributing to these secondary peaks are exclusively found in the neighborhood of GBs and T.Js.

With further deformation the mean value of $\|\boldsymbol{M}\|$ appears to converge to a threshold. This is because couple stresses are generated as a direct consequence of local perturbations of Cauchy stresses and, therefore, depend on 


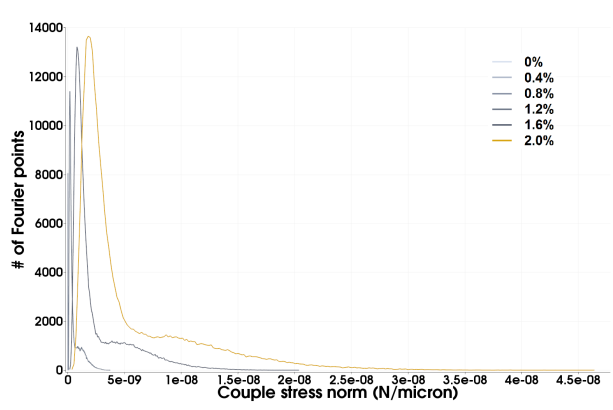

(a) Linear

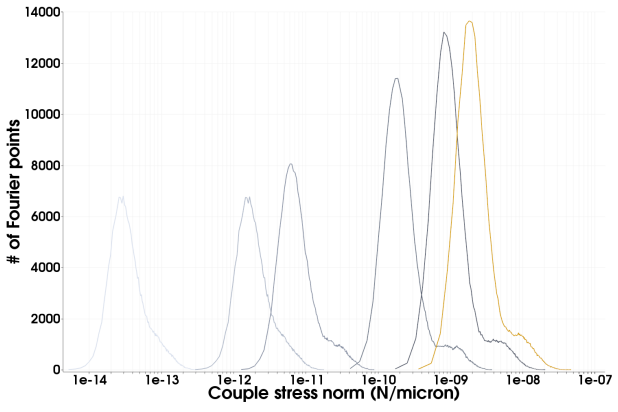

(b) Semi-log

Figure 7: Couple stress norm $\|\boldsymbol{M}\|$ histograms with (a) linear and (b) semi-logarithmic axes for the $20 \mathrm{~nm}$ microstructure loaded from $E_{22}=0 \%$ to $2 \%$ strain at $\dot{E}_{22} 1 / \mathrm{s}$. Each histogram is computed using 256 bins, taking values from every Fourier point in the domain.

their evolution; as the latter converges to a threshold value, the former follows it. At the end of loading, the right shoulder evolves into multiple shorter peaks which appear to converge into a distinct secondary peak. The incompatibility of slip across the GBs results in accumulation of plastic curvature in their vicinity resulting in the formation of polar dislocations and disclinations (shown in figure 8). In addition to elastic curvatures due to lattice mismatch across the GB, the generation of these defects contributes to the local elastic curvatures and hence the couple stresses. These couple stresses contribute to the formation of the second peak. For the cases where VM Cauchy stress approaches its saturation value, for example microstructures loaded at $10^{-3} / \mathrm{s}$, the increase in intensity of the primary peak in $\|\boldsymbol{M}\|$ (not shown here) is found to reduce and the secondary peak tends to assimilate into the primary peak forming the right shoulder originally seen in the elastic regime. This indicates that with larger plastic deformation the grain interiors observe an increase in magnitude of couple stresses and curvatures faster than those in the vicinity of GBs where Cauchy stresses have saturated.

Figure 8(a) shows the comparison of norm of density of polar dislocations $\|\boldsymbol{\alpha}\|$ generated for the $20 \mathrm{~nm}$ microstructure loaded to $E_{22}=2 \%$ strain at $10^{-3} / \mathrm{s}$. The largest percentage difference in $\|\boldsymbol{\alpha}\|$ predicted by both models is $16.667 \%$, indicating that plastic strains have a dominant contribution to polar dislocation density over plastic curvatures. However, this difference 
is non-negligible and noting that CSEVP DFT-I predicts a lower $\|\boldsymbol{\alpha}\|$ than EVP FFT, it underlines the importance of plastic curvatures in reducing long range stress inducing polar dislocations with the formation of polar disclinations. Figure 8(b) shows the polar disclination density generated due to the accumulation of plastic curvatures (from equation (11)). As can be expected, local maxima in polar disclination densities exist in the vicinity of GBs. Non-zero polar disclination densities are also found in grain interiors. This indicates that sub-grain boundaries must be forming due to plastic curvature accumulation in grain interiors. This result in combination with the macroscopic response shown in figure 5 strongly indicate that disclination nucleation is a possible mechanism to accommodate stresses in the medium.

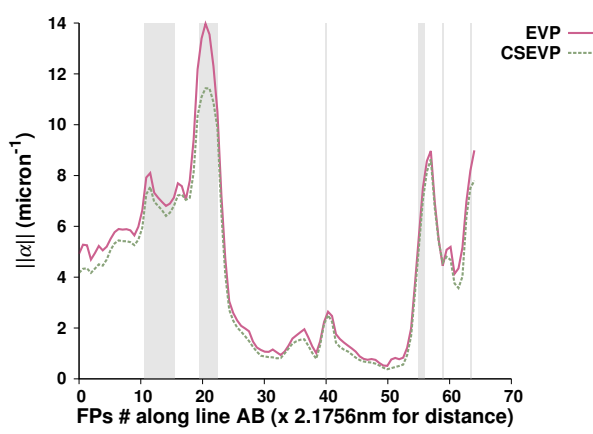

(a) $\|\boldsymbol{\alpha}\|$

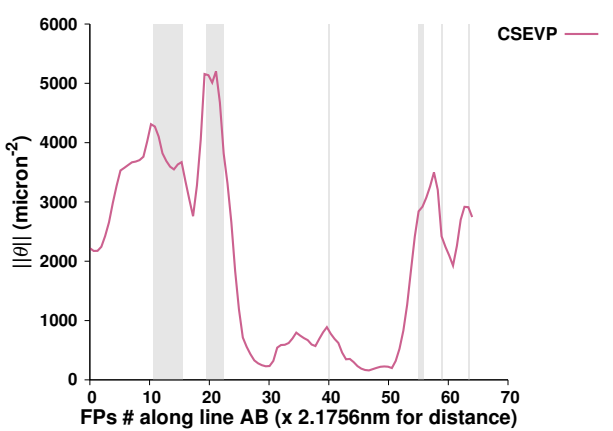

(b) $\|\boldsymbol{\theta}\|$

Figure 8: (a) Comparison of norm of polar dislocation density $\|\boldsymbol{\alpha}\|$ predicted by CSEVP DFT-I and EVP FFT, and (b) norm of polar disclination density $\|\boldsymbol{\theta}\|$ predicted by CSEVP DFT-I for the $20 \mathrm{~nm}$ microstructure loaded to $E_{22}=2 \%$ at $10^{-3} / \mathrm{s}$.

\subsection{Role of initial GB curvatures on local and effective mechanical response of nc microstructures}

In the previous section it was seen that accumulation of plastic strain and curvature results in the increase of polar defects in vicinity of GBs and TJs. In this section, the effect of presence of these polar defects, captured through initial curvatures, on the mechanical response of nc materials is studied. The study is performed for the three configurations $t_{0}, t_{1}$ and $t_{2}$ described in section 5 . 


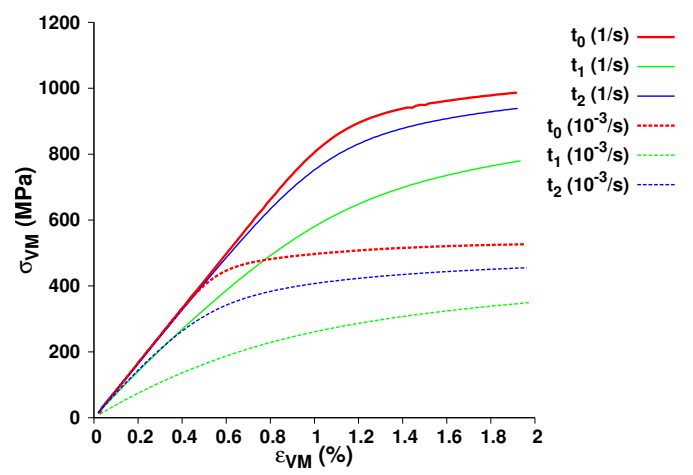

(a) $20 \mathrm{~nm}$

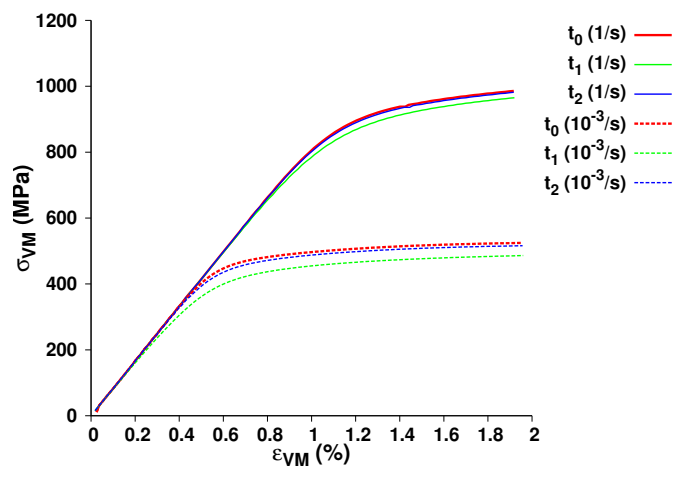

(b) $40 \mathrm{~nm}$

Figure 9: Comparison of VM Cauchy stress vs equivalent strain curves predicted for $t_{0}$, $t_{1}$ and $t_{2}$ microstructures with average grain size (a) $20 \mathrm{~nm}$ and (b) $40 \mathrm{~nm}$ for strain rates $\dot{E}_{22}=0.001 / s$ and $\dot{E}_{22}=1 / s$.

Figure 9(a) and (b) show the comparison between VM Cauchy stress and equivalent strains obtained for the $t_{0}, t_{1}$ and $t_{2}$ configurations of $20 \mathrm{~nm}$ and $40 \mathrm{~nm}$ microstructures, respectively, for two loading rates. For all the cases studied, the softening effect is the highest for the $t_{1}$ configuration followed by $t_{2}$ while the $t_{0}$ configuration has negligible softening. This is because the $t_{1}$ configuration, which has a three times higher magnitude of initial curvatures in comparison with $t_{2}$, results in the generation of local Cauchy stresses with larger magnitudes. This results in earlier initiation of plasticity in the $t_{1}$ configuration. The macroscopic effect of this is a softer response for the $t_{1}$ configuration in comparison with the $t_{2}$ configuration. Furthermore, magnitudes of initial curvatures for both $t_{1}$ and $t_{2}$ configurations are two times larger in the $20 \mathrm{~nm}$ microstructure than in the $40 \mathrm{~nm}$ microstructure. Hence, for both $t_{1}$ and $t_{2}$, the macroscopic response is softer for the $20 \mathrm{~nm}$ microstructure in comparison with the $40 \mathrm{~nm}$ microstructure. Since both microstructures are loaded to the same macroscopic strain but at different loading rates, the relaxation of local Cauchy stresses generated from initial curvatures occurs at different rates for each configuration. This results in a variation in the spread in macroscopic Cauchy stress saturation values between $t_{0}, t_{1}$ and $t_{2}$ configurations for the same microstructure loaded at different rates. While for the $t_{0}$ configuration, the strain rate sensitivity effect does not vary with grain size, there are some variations observed for 
the $t_{1}$ and $t_{2}$ configurations. For the 20nm and 40nm microstructure the difference in macroscopic Cauchy stress at $E_{22}=2 \%$ is $430.4 \mathrm{MPa}$ and 478.8 MPa, respectively. From these results, it can be deduced that the spread in macroscopic Cauchy stress saturation values increases with decreasing grain size and strain rate.

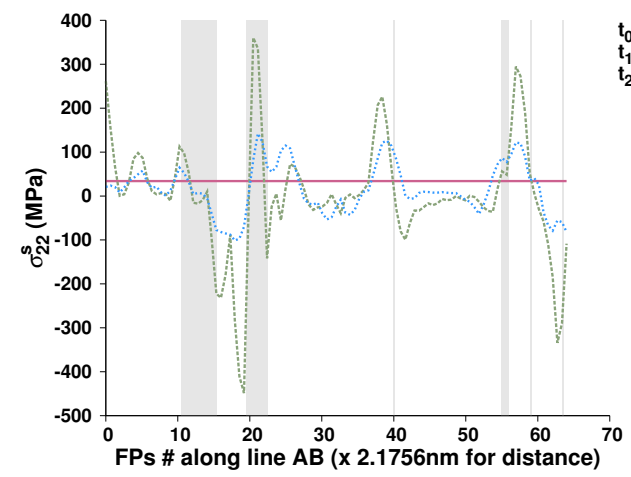

(a) $20 \mathrm{~nm}$

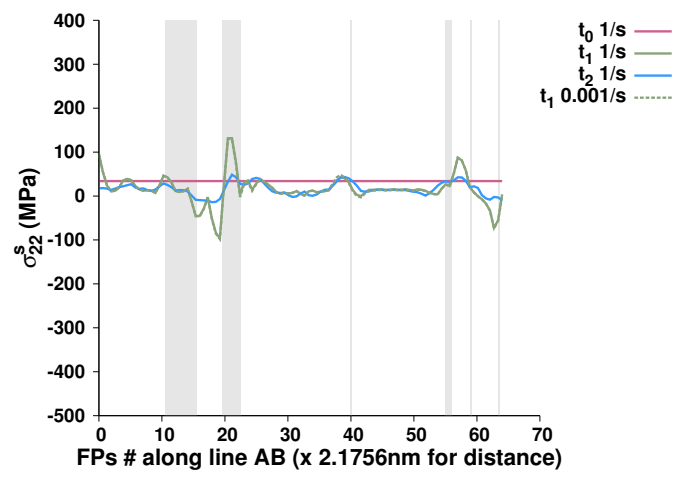

(b) $40 \mathrm{~nm}$

Figure 10: Plot-over-line $\mathrm{AB}$ from figure 3 of Cauchy stress $\sigma_{22}^{s}$ for $t_{0}, t_{1}$ and $t_{2}$ loaded at $1 / \mathrm{s}$ along with $t_{1}$ loaded at $0.001 / \mathrm{s}$ at the initial time step for (a) $20 \mathrm{~nm}$ and (b) $40 \mathrm{~nm}$ microstructures.

Inhomogeneous distribution of eigencurvatures results in generation of local fluctuations in couple stresses which in turn result in Cauchy stress fluctuations in order to maintain the local equilibrium. Figures 10(a) and (b) show the plot over line $\mathrm{AB}$ from figure 3 of $\sigma_{22}$ at the first time step for $t_{0}, t_{1}$ and $t_{2}$ configurations loaded at $1 / s$ and $0.001 / s$. For a given inter Fourier point distance, the magnitude of Cauchy stress fluctuations is strongly influenced by the magnitude and distribution of GB curvatures. For both the loading rates, these fluctuations have the highest amplitude for the $t_{1}$ configuration. Furthermore, with decreasing inter Fourier point distance, or average grain size, the role of strain rate on the local Cauchy stress fluctuations becomes increasingly important.

Figure 11 compares the grain-averaged plastic shear from a randomly selected grain during loading (the same grain is used from all configurations). Without initial curvatures, the plastic activity is governed by the crystallographic orientation of the grain as seen in figure 11(a) for the $t_{0}$ configuration. However, figures 11(b) and (c) show an earlier initiation of plasticity in the 


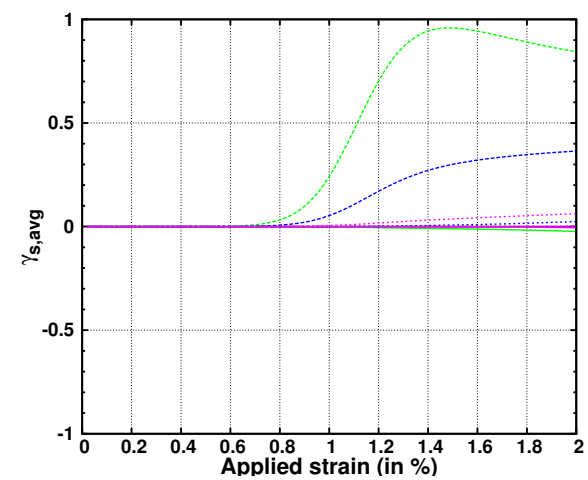

(a) $t_{0}$
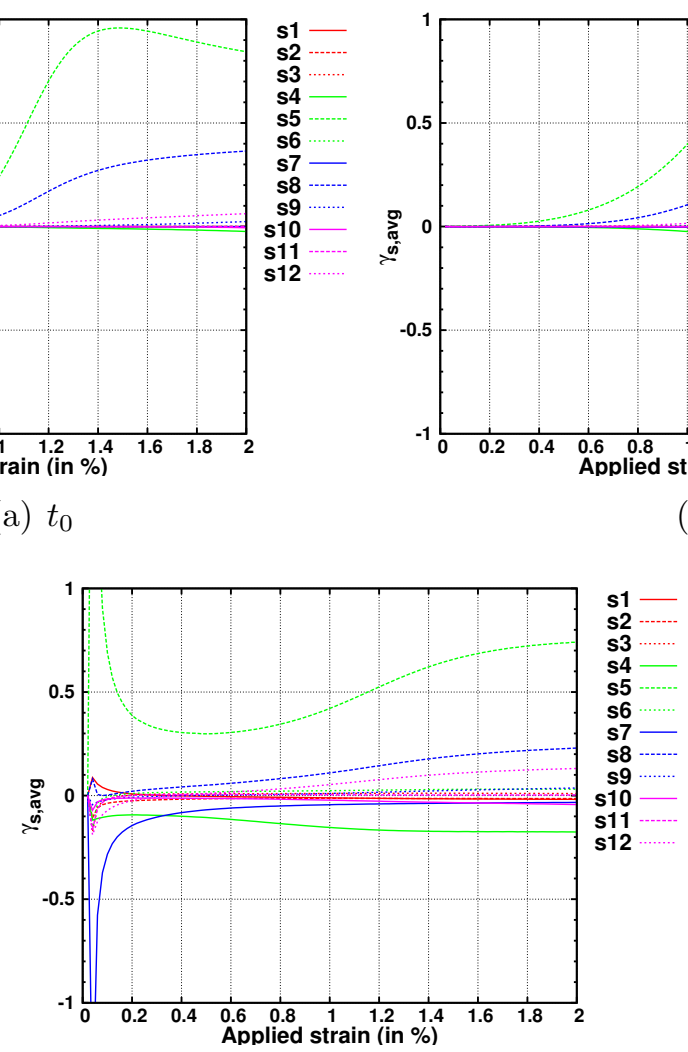

(c) $t_{1}$

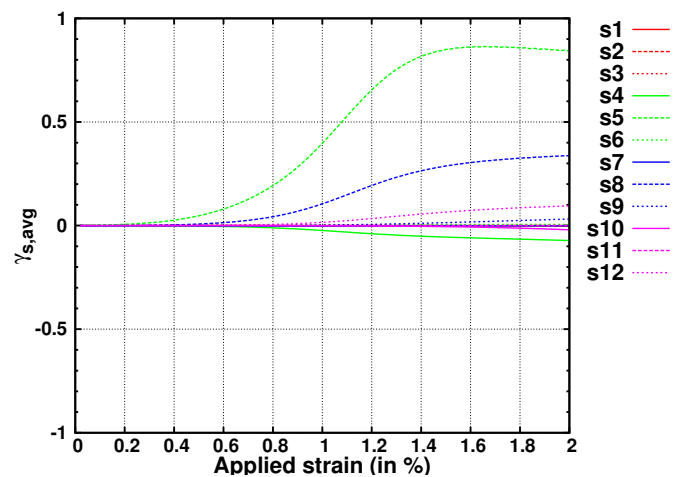

(b) $t_{2}$

Figure 11: Averaged plastic shear from a randomly chosen grain in the $20 \mathrm{~nm}$ microstructure for the $t_{0}, t_{2}$ and $t_{1}$ configurations.

$t_{1}$ and $t_{2}$ configurations due to the Cauchy stresses generated from the initial curvatures. Moreover, for the $t_{1}$ configuration, additional slip systems are activated. Note that the amount of plastic shear generated is governed by the power law in equation (17). This law is derived assuming continuous shearing events in large crystals and may not be appropriate to describe discrete dislocation or GB plasticity events that are typically observed in nc materials [78, 79].

Figure 12(a) and (b) shows the plot over line AB from figure 3 of $\sigma_{22}$ at $0.8 \%$ and $2 \%$ applied strains, respectively, at a rate of $1 / \mathrm{s}$ for the $20 \mathrm{~nm} t_{0}, t_{1}$ and $t_{2}$ configurations. In conjunction with figure 11 , at $0.8 \%$ applied strain, 


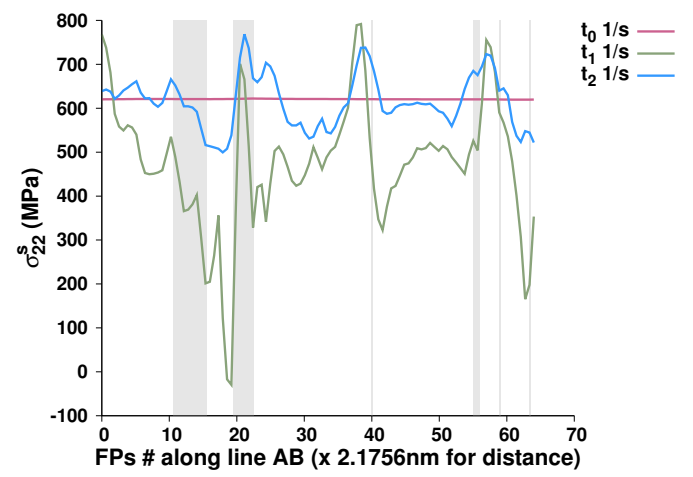

(a) At $E_{22}=0.8 \%$

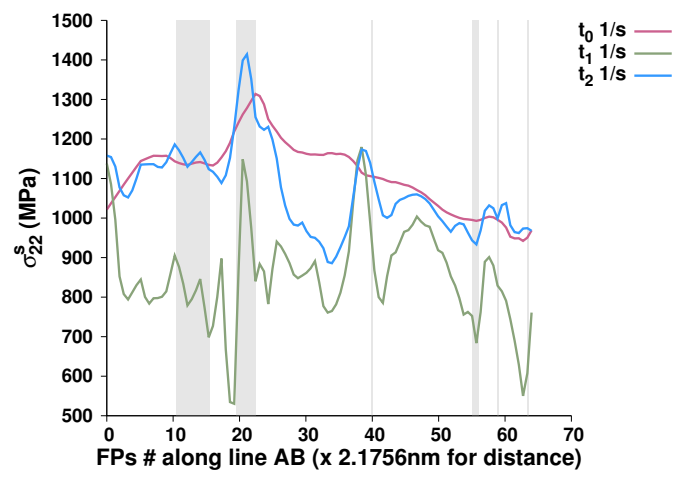

(b) At $E_{22}=2.0 \%$

Figure 12: Plot-over-line $\mathrm{AB}$ from figure 3 of Cauchy stress $\sigma_{22}^{s}$ for $t_{0}, t_{1}$ and $t_{2}$ loaded at $1 / \mathrm{s}$ to (a) $E_{22}=0.8 \%$ and (b) $E_{22}=2.0 \%$ strain for the $20 \mathrm{~nm}$ microstructure.

plastic accommodation for $t_{1}$ and $t_{2}$ is found to be governed dominantly by the local Cauchy stress fluctuations induced via initial GB curvatures. At $2 \%$ strain, the Cauchy stress evolution for $t_{2}$ configuration is found to be closer to the plastic anisotropic response. However, for the $t_{1}$ configuration, Cauchy stresses persistently evolve as a function of GB neighborhood. This is also reflected in the different magnitudes of slip activity for the three configurations in figure 11.

For the $20 \mathrm{~nm} t_{1}$ configuration, the large local Cauchy stresses generated result in an unstable initial nc microstructure configuration. Such magnitudes and distributions of polar defects (captured through eigencurvatures) are unlikely to occur in these materials. One way to stabilize the response of the $20 \mathrm{~nm} t_{1}$ configuration is to take different fractions of initial curvatures as the negative eigencurvatures as discussed in section 5.1. The macroscopic VM Cauchy stress vs strain response, local Cauchy stress plot-over-line AB at $2 \%$ applied strain and slip activity for four different fractions of the initial curvature for $20 \mathrm{~nm} t_{1}$ configuration are shown in figure 13 . As the magnitude of eigencurvatures decreases, the local and macroscopic mechanical response converges towards the plastic anisotropic response. Interestingly, the macroscopic response of $20 \mathrm{~nm} t_{2}$ configuration (figure 9) and average shear evolution (figure 11) follow a similar trend as the $t_{1} 0.4 \kappa^{\text {ini }}$ configuration, however, the local Cauchy stress (figure 12) evolution is different. This indicates that the magnitude and distribution of initial curvatures is crucial 


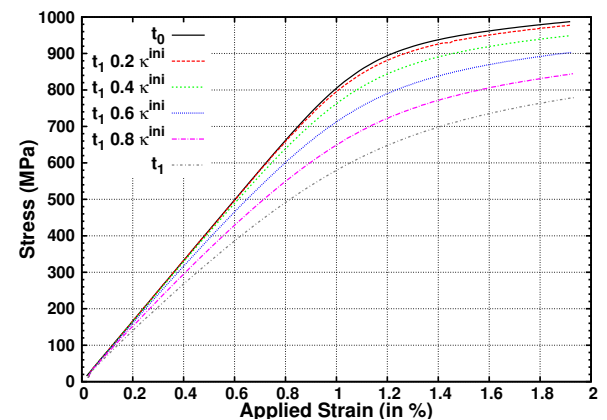

(a) VM Cauchy stress vs strain

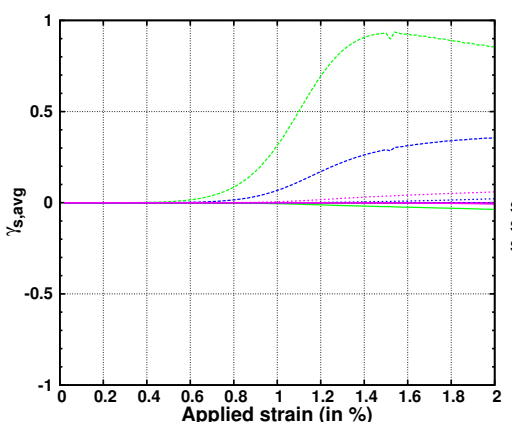

(c) Average shear for $0.2 \kappa^{i n i}$

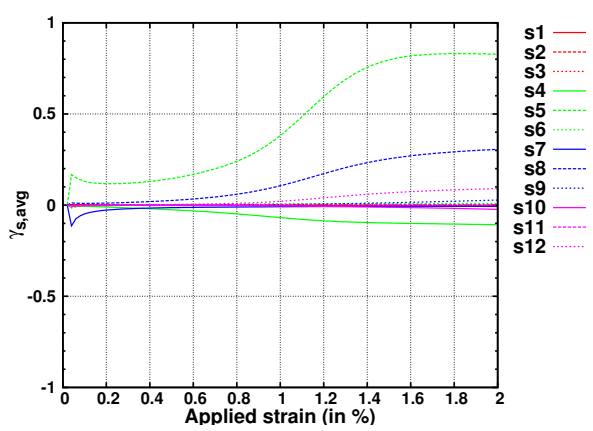

(e) Average shear for $0.6 \kappa^{i n i}$

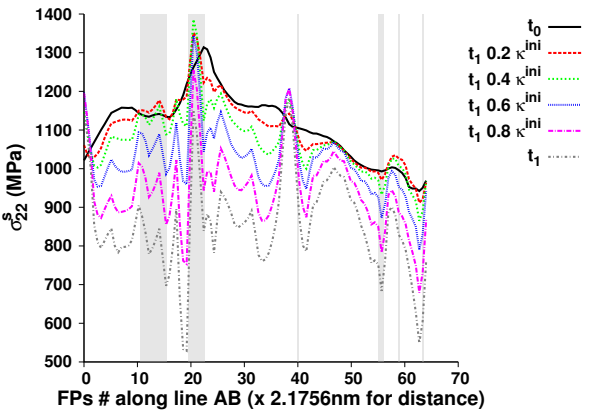

(b) $\sigma_{22}^{s}$ plot-over-line
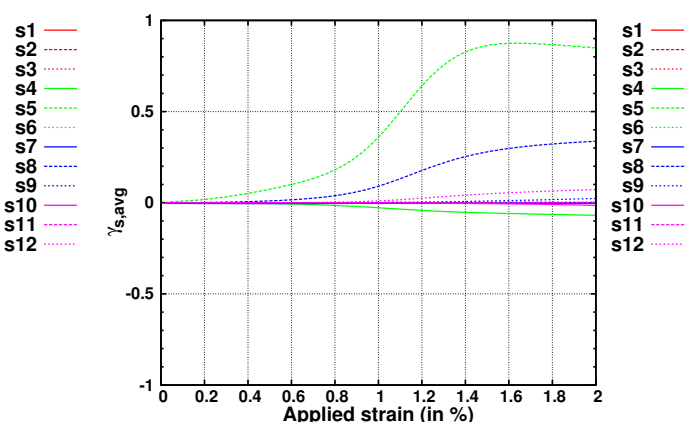

(d) Average shear for $0.4 \kappa^{i n i}$

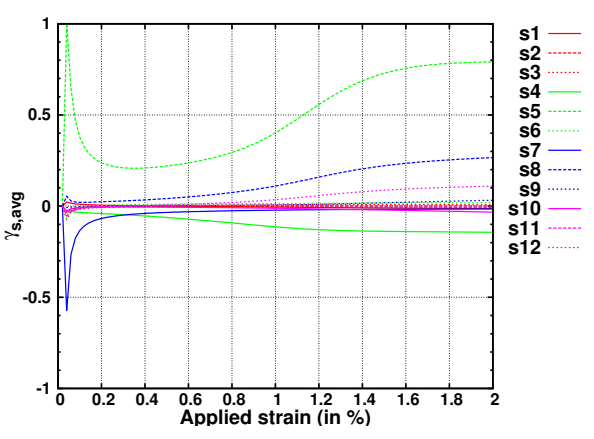

(f) Average shear for $0.8 \kappa^{i n i}$

Figure 13: Comparison of different fraction of initial curvatures taken as eigencurvatures for the $20 \mathrm{~nm} t_{1}$ configuration loaded at $\dot{E}_{22}=1 / \mathrm{s}$ : (a) VM Cauchy stress vs strain response (b) Plot-over-line $\mathrm{AB}$ from figure 3 of Cauchy stress $\sigma_{22}^{s}$ at $E_{22}=2 \%$. Average plastic shear in a randomly chosen grain for eigencurvatures equal negative of (c) $0.2 \kappa^{\text {ini }}$, (d) $0.4 \kappa^{i n i}$, (e) $0.6 \kappa^{i n i}$ and (f) $0.8 \kappa^{i n i}$ 
in capturing the local stress state of the medium that governs the activation of plasticity mechanisms in nc materials.

Figure 14(a) shows the polar dislocation densities for the $t_{0}, t_{1}$ and $t_{2}$ configurations. Plastic strains are found to have a dominant contribution to the polar dislocation densities in comparison with plastic curvatures. This is also indicated through the close match in the trends, although the magnitudes of these densities are different for the three microstructure configurations (highest to lowest in the order $t_{1}, t_{2}$ and $t_{0}$ ). This indicates that plastic anisotropy governs the evolution of polar dislocation densities. This match in the trends indicates that plastic anisotropy governs the evolution of polar dislocation densities. This is to be expected since the plastic strain evolution is modeled as a function of the Schmid tensor in equation (17), and as mentioned earlier it may not be appropriate for modeling nc plasticity.

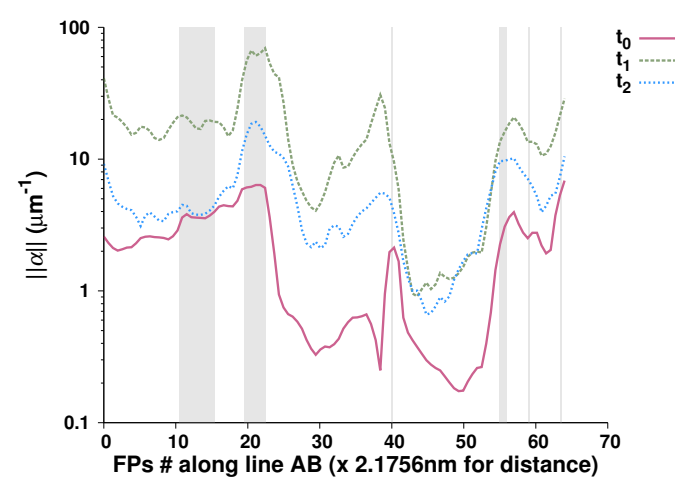

(a) $\|\boldsymbol{\alpha}\|\left(\mu m^{-1}\right)$

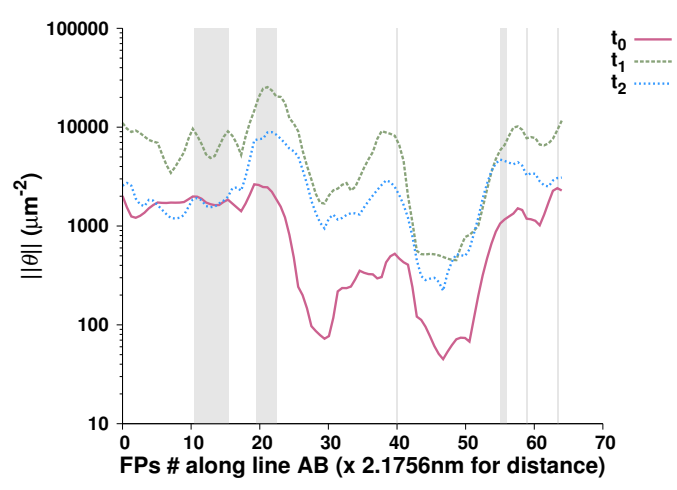

(b) $\|\boldsymbol{\theta}\|\left(\mu m^{-2}\right)$

Figure 14: Log normal plot-over-line AB from figure 3 of (a) $\|\boldsymbol{\alpha}\|\left(\mu m^{-1}\right)$ and (b) $\|\boldsymbol{\theta}\|$ $\left(\mu^{-2}\right)$ for $t_{0}, t_{1}$ and $t_{2}$ loaded to $2 \%$ strain at a rate of $1 / \mathrm{s}$ for the $20 \mathrm{~nm}$ microstructure.

Plastic curvatures generated due to dislocation activity result in the generation of polar disclination densities. These are a consequence of dislocation interactions with GBs; note here that these are not numerical artifacts such as the ones arising from prescribing initial curvatures. Comparing figures 9, 12 and 14(b), it is found that the decrease in average values of Cauchy stresses for the $t_{1}, t_{2}$ and $t_{0}$ microstructure is related to an increase in both polar dislocation and disclination densities. Combined nucleation of polar disclinations and dislocations could be a potential mechanism to accommodate plasticity in nc materials. 


\section{Discussion}

\subsection{Curvature based description of $G B s$}

In applications of classical continuum crystal plasticity models to nc materials, GB interfaces were treated as a separate phase from grain interiors. For example, following a pressure dependent response based on Drucker type yield criterion [27] or Mohr Coulomb type yield criterion [28, 80]. In the present work, GBs and grain interiors are considered as the same phase respecting the same higher order continuum equilibrium and constitutive laws.

The curvature based characterization of the GB has the potential for reinforcing the synergy between FFT based approaches and orientation mapping techniques which is why they were initially designed. The initial curvatures can be taken as an input directly from technique based on electron back scattering diffraction $[72,73]$. Prior to this, however, it is important to tackle the problem of appropriately computing the magnitude and distribution of these curvatures in nc materials.

Mesoscale GBs are typically treated as singular interfaces and are traditionally modeled with the help of surface dislocations and disclinations [81-83]. In reality, however, the transition from one grain orientation to another is a gradual change across a region of finite thickness. Capturing the non-singularity of the GB region requires surrendering the concept of surface dislocations and disclinations by fulfilling tangential continuity conditions on the elastic/plastic strains and curvatures and their rates [41] along the GB interface. Treating these tangential continuity conditions, however, is beyond the scope of the present work. Therefore, characterizing GB interfaces using the technique described in section 3.4 entails the presence of surface defects.

\subsection{GB structure based local stresses and slip activity}

Characterizing the initial microstructure using GB curvatures results in the generation of local Cauchy stresses in the vicinity of these GBs. These stresses are different from those generated due to the crystallographic orientation of grains under the action of applied loads. As a consequence, dislocations may nucleate on slip systems which are crystallographically not favored with respect to the applied load. Furthermore, the magnitude of these stresses depends on the magnitude of initial GB curvatures. This could help explain why the nucleation and propagation of dislocations occurs in a very few grains within a grain family of the same crystallographic orientation. 
The macro scale "softening" effect predicted by the present model is also a function of the magnitude and distribution of inital GB curvature. As the grain size increases (i.e. the inter Fourier point distance is increased while keeping their number constant), the magnitude of initial GB curvatures decreases. Consequently the magnitude of local Cauchy stresses are reduced. Macroscopically, this results in a lower strain rate sensitivity and reduced softening effect. In other words, the role of curvatures in determining the local and macroscopic stress state becomes crucial when the average grain size of nc materials are of the order of the critical size below which the inverse Hall-Petch effect is observed [6, 7, 12-14].

\subsection{Prospective work}

In the present work, the importance of characterizing initial microstructures using lattice curvature was highlighted, however, there are several other important factors that need to be addressed before expecting a reliable prediction of the mechanical behavior of nc materials. A few of the crucial ones are listed in the following:

(1) Couple stress constitutive relationship and incompatibility: the influence of elastic curvatures and couple stresses is highly dependent on the elastic constant $\boldsymbol{A}$. In this work, it is assumed to be isotropic linear elastic [57] and estimated as $\boldsymbol{A}=G b^{2}$. However, an accurate value for this constant is yet to be determined. Increasing (or decreasing) the magnitude of $\boldsymbol{A}$ from $G b^{2}$, while using the same magnitude of initial curvature, will increase (or decrease) the magnitudes of Cauchy stresses generated. Thus, producing an increased (or decreased) "softening" effect.

In order to capture the non-local behavior of GBs, it is important to first appropriately distinguish between compatible and incompatible components of elastic curvatures and account for the cross terms in the elastic constitutive relationship [57].

(2) Boundary conditions: The CSEVP DFT-I model allows prescribing higher order BCs in the form of curvature and couple stresses along with the Cauchy stress and strain BCs. These can be used to study the effect of bending/torsion along with complex mixed boundary conditions on the local and effective response of polycrystalline materials. However, special care must be taken when prescribing higher order boundary conditions on the periodic microstructure. For example, the displacements resulting from prescribing curvatures on the boundaries of a microstructure are non-homogeneous and 
their consequence on periodic representative microstructures needs to be better understood [84].

(3) Mechanism based plasticity: the transport of dislocations and disclinations should be incorporated to study interplay between diffusion related and stress driven GB and dislocation based mechanisms. Prior to this, however, the physical understanding of disclination mobility needs to be addresssed.

(4) Disclination based understanding of GB mechanisms: The CSEVP DFT-I approach allows explicit kinematic characterization of GBs using disclinations in addition to dislocations. Its application in the present work has highlighted that the nucleation of both polar disclinations along with dislocations could result in further lowering of Cauchy stresses than from just dislocation based model. At the fine scale, Fressengeas and co-workers [43, 44] have shown that capturing the appropriate dynamics of GBs requires going beyond the conventional dislocation based approach to include disclinations. Loosely supporting these simulations are a scant collection of experimental observations of disclinations in the vicinity of GBs and TJs $[38,85]$. The disclination based characterization of GBs needs to be rigorously pursued.

(5) Material specific plasticity: Mechanisms based on material properties such as the stacking fault energy, should be appropriately incorporated. For example, the quantized crystal plasticity model of Li et al. [78] can be incorporated for the initial stages of deformation in some nc FCC materials, while for later stages when dynamic grain growth is activated in some materials, the Schmid law criterion can be used for grains above a certain grain size [79].

(6) Incorporating strain gradient plasticity: The CSEVP DFT-I model falls in a general category of higher order crystal plasticity models [71, 86-91] and should be extended to account for strain gradient plasticity effects in order to appropriately capture the local microstructure, polar defect content and hardening behavior of nc materials.

However, from a kinematic point of view the purely strain gradient plasticity framework is unable to handle curvature incompatibilities at the interface. These can be treated using a curvature based model such as the one proposed in the present work or a generalized distortion gradient plasticity model such as the proposed by Acharya and Fressengeas [92] that accounts for both strain and curvature incompatibilities. 


\section{Conclusion}

In the present work, the CSEVP DFT-I model is developed to highlight the role of lattice curvatures on the meso and macro scale mechanical response of nc materials. The CSEVP problem involves solving a couple stress continuum based elasto-viscoplastic problem for periodic RVEs. A couple stress based Green's function formulation is proposed to analytically solve this problem. Its numerical formulation uses the DFT-I technique which is a combination of continuous and discrete FFT approaches. This is developed to stabilize the Gibbs oscillations arising from discontinuous curvature fields.

The CSEVP DFT-I model is then applied to nc RVEs of different grain sizes. The GBs in the initial microstructure are either treated as singular interfaces or characterized using a curvature based approach. The main findings from this study are: (1) Elastic curvatures accommodate a part of the Cauchy stresses generated during loading, although elastic and plastic strains play a dominant role. (2) At the meso scale, GB lattice curvatures could lead to the generation of localized stresses driving plastic activity on slip systems that are crystallographically not favored under the action of applied stresses. (3) At the macroscale, this results in the size and strain rate dependent "softening" effect, i.e. inverse Hall-Petch effect that is characteristic of fine grained nc materials. (4) The combined effect of generating dislocations and disclinations could result in significant lowering of local stresses, and capturing these effects requires a higher order model accounting for curvatures and couple stresses in incompatible media.

\section{Acknowledgements}

M. V. Upadhyay, L. Capolungo, V. Taupin and C. Fressengeas would like to acknowledge the support of ANR (Agence National de la Recherche) under Grant ANR-11-JS09-007-01, NanoMec.

\section{References}

[1] H. Gleiter, Nanocrystalline Materials, Progress in Materials Science 33 (1989) $223-315$.

[2] H. Gleiter, Nanostructured Materials: Basic Concepts and Microstructure, Acta Materialia 48 (2000) 1 - 29. 
[3] G. Hasson, J.-Y. Boos, I. Herbeuval, M. Biscondi, C. Goux, Theoretical and experimental determinations of grain boundary structures and energies: Correlation with various experimental results, Surface Science 31 (1972) $115-137$.

[4] D. Wolf, M. Kluge, Relationship between shear resistance and local atomic structure at grain boundaries in FCC metals, Scripta Materialia 24 (1990) 907-914.

[5] M. Tschopp, G. Tucker, D. McDowell, Structure and Free Volume of $<110>$ Symmetric Tilt Grain Boundaries with the E Structural Unit, Acta Materialia 55 (2007) 3959 - 3969.

[6] K. S. Kumar, H. V. Swygenhoven, S. Suresh, Mechanical Behavior of Nanocrystalline Metals and Alloys, Acta Materialia 51 (2003) 5743 5774 .

[7] M. Dao, L. Lu, R. J. Asaro, J. T. M. D. Hosson, E. Ma, Toward a Quantitative Understanding of Mechanical Behavior of Nanocrystalline metals, Acta Materialia 55 (2007) 4041 - 4065.

[8] E. O. Hall, The Deformation and Ageing of Mild Steel: III Discussion of Results, Proceedings of the Physical Society B 64 (1951) 747-753.

[9] N. J. Petch, The cleavage strength of polycrystals, Journal of the Iron and Steel Institute 174 (1953) 25-28.

[10] A. S. Khan, J. Liu, J. W. Yoon, R. Nambori, Strain rate effect of high purity aluminum single crystals: Experiments and simulations, International Journal of Plasticity 67 (2015) 39-52.

[11] J. Liu, A. S. Khan, L. Takacs, C. S. Meredith, Mechanical behavior of ultrafine-grained/nanocrystalline titanium synthesized by mechanical milling plus consolidation: Experiments, modeling and simulation, International Journal of Plasticity 64 (2015) 151-163.

[12] J. Schiøtz, K. W. Jacobsen, A Maximum in the Strength of Nanocrystalline Copper, Science 301 (2003) 1357 - 1359.

[13] K. J. V. Vliet, S. Tsikata, S. Suresh, Model Experiments for direct Visualization of Grain Boundary Deformation in Nanocrystalline Metals, Applied Physics Letters 83 (2003) 1441 - 1443. 
[14] C. A. Schuh, T. G. Nieh, T. Yamasaki, Hall-Petch Breakdown Manifested in Abrasive Wear Resistance of Nanocrystalline Nickel, Scripta Materialia 46 (2002) $735-740$.

[15] J. Carsley, J. Ning, W. Milligan, S. Hackney, E. Aifantis, A simple, mixture-based model for the grain-size dependence of strength in nanophase metals, Nanostructured Materials 5 (1995) 441-448.

[16] D. Konstantinidis, E. Aifantis, On the anomalous hardness of nanocrystalline materials, Nanostructured Materials 10 (1998) 1111-1118.

[17] H. Kim, Y. Estrin, M. Bush, Plastic deformation behavior of fine grained materials, Acta Materialia 48 (2000) 493-504.

[18] H. Fu, D. Benson, M. Meyers, Analytical and computational description of effect of grain size on yield stress of metals, Acta Materialia 49 (2001) $2567-2582$.

[19] D. Benson, H. Fu, M. Meyers, On the effect of grain size on yield stress: extension into nanocrystalline domain, Materials Science and Engineering: A 319-321 (2001) 854-861.

[20] H. Fu, D. Benson, M. Meyers, Computational description of nanocrystalline deformation based on crystal plasticity, Acta Materialia 52 (2004) 4413-4425.

[21] H. Kim, Y. Estrin, Phase mixture modelling of the strain rate dependent mechanical behavior of nanostructured materials, Acta Materialia 53 (2005) 765-772.

[22] L. Capolungo, M. Cherkaoui, J. Qu, A self-consistent model for the inelastic deformation of nanocrystalline materials, Journal of Engineering Materials and Technology - Transactions of the ASME 127 (2005) 400-407.

[23] L. Capolungo, C. Jochum, M. Cherkaoui, J. Qu, Homogenization method for strength and inelastic behavior of nanocrystalline materials, International Journal of Plasticity 21 (2005) 67-82.

[24] Y. Wei, L. Anand, Grain-boundary sliding and separation in polycrystalline metals: application to nanocrystalline fcc metals, Journal of the Mechanics and Physics of Solids 52 (2004) 2587-2616. 
[25] B. Zhu, R. Asaro, P. Krysl, R. Bailey, Transition of deformation mechanisms and its connection to grain size distribution in nanocrystalline metals, Acta Materialia 53 (2005) 4825-4838.

[26] Y. Wei, H. Gao, An elastic-viscoplastic model of deformation in nanocrystalline metals based on coupled mechanisms in grain boundaries and grain interiors, Materials Science and Engineering: A 478 (2008) 16-25.

[27] B. Jiang, G. Weng, A generalized self-consistent polycrystal model for the yield strength of nanocrystalline materials, Journal of the Mechanics and Physics of Solids 52 (2004) 1125-1149.

[28] R. Lebensohn, E. Bringa, A. Caro, A viscoplastic micromechanical model for the yield strength of nanocrystalline materials, Acta Materialia 55 (2007) 261-271.

[29] K. Aifantis, J. Willis, The role of interfaces in enhancing the yield strength of composites and polycrystals, Journal of the Mechanics and Physics of Solids 53 (2005) 1047-1070.

[30] M. E. Gurtin, A finite-deformation, gradient theory of single-crystal plasticity with free energy dependent on densities of geometrically necessary dislocations, International Journal of Plasticity 24 (2008) 702-725.

[31] K. Aifantis, A. Konstantinidis, Hall-Petch revisited at the nanoscale, Materials Science and Engineering: B 163 (2009) 139-144.

[32] K. Aifantis, On the gradient approach - Relation to Eringen's nonlocal theory, International Journal of Engineering Science 49 (2011) 13671377.

[33] H.-K. Kim, S.-I. Oh, An interfacial energy incorporated couple stress crystal plasticity and the finite element simulation of grain subdivision, Journal of the Mechanics and Physics of Solids 60 (2012) 1815-1841.

[34] G. Z. Voyiadjis, D. Faghihi, Y. Zhang, A theory for grain boundaries with strain-gradient plasticity, International Journal of Solids and Structures 51 (2014) 1872-1889. 
[35] Y. Aoyagi, T. Tsuru, T. Shimokawa, Crystal plasticity modeling and simulation considering the behavior of the dislocation source of ultrafinegrained metal, International Journal of Plasticity 55 (2014) 43-57.

[36] P. van Beers, V. Kouznetsova, M. Geers, M. Tschopp, D. McDowell, A multiscale model of grain boundary structure and energy: From atomistics to a continuum description, Acta Materialia 82 (2015) 513-529.

[37] P. van Beers, V. Kouznetsova, M. Geers, Grain boundary interfacial plasticity with incorporation of internal structure and energy, Mechanics of Materials doi:http://dx.doi.org/10.1016/j.mechmat.2015.01.010.

[38] H. Rösner, C. Kübel, Y. Ivanisenko, L. Kurmanaeva, S. V. Divinski, M. Peterlechner, G. Wilde, Strain mapping of a triple junction in nanocrystalline Pd, Acta Materialia 59 (2011) 7380-7387.

[39] F. Di Gioacchino, J. Quinta da Fonseca, An experimental study of the polycrystalline plasticity of austenitic stainless steel, International Journal of Plasticity 74 (2015) 92-109.

[40] J. L. W. Carter, J. M. Sosa, P. A. Shade, H. L. Fraser, M. D. Uchic, M. J. Mills, The potential link between high angle grain boundary morphology and grain boundary deformation in a nickel-based superalloy, Materials Science and Engineering A 640 (2015) 280-286.

[41] C. Fressengeas, V. Taupin, M. Upadhyay, L. Capolungo, Tangential Continuity of Elastic/Plastic Curvature and Strain at Interfaces, International Journal of Solids and Structures 49 (2012) 2660 - 2667.

[42] M. Upadhyay, L. Capolungo, V. Taupin, C. Fressengeas, Grain boundary and triple junction energies in crystalline media: A disclination based approach, International Journal of Solids and Structures 48 (2011) 31763193.

[43] V. Taupin, L. Capolungo, C. Fressengeas, A. Das, M. Upadhyay, Grain Boundary Modeling using an Elasto-Plastic Theory of Dislocation and Disclination Fields, Journal of the Mechanics and Physics of Solids 61 (2013) $370-384$. 
[44] C. Fressengeas, V. Taupin, L. Capolungo, Continuous Modeling of the Structure of Symmetric Tilt Boundaries, International Journal of Solids and Structures 51 (2014) $1434-1441$.

[45] V. Taupin, L. Capolungo, C. Fressengeas, Disclination Mediated Plasticity in Shear-Coupled Boundary Migration, International Journal of Plasticity 53 (2014) 179 - 192.

[46] V. Taupin, L. Capolungo, C. Fressengeas, M. V. Upadhyay, B. Beausir, A mesoscopic theory of dislocation and disclination fields for grainboundary mediated crystal plasticity, International Journal of Solids and Structures 71 (2015) 277-290.

[47] H. Moulinec, P. Suquet, A Fast Numerical Method for Computing the Linear and Nonlinear Properties of Composites, Comptes Rendus de l'Académie des Sciences Paris II 318 (1994) 1417 - 1423.

[48] H. Moulinec, P. Suquet, A Numerical Method for Computing the Overall Response of Nonlinear Composites with Complex Microstructure, Computer Methods in Applied Mechanics and Engineering 157 (1998) 69 94.

[49] R. Lebensohn, N-site Modeling of a 3D Viscoplastic Polycrystal using Fast Fourier Transform, Acta Materialia 49 (2001) 2723 - 2737.

[50] R. Lebensohn, A. Kanjarla, P. Eisenlohr, An Elasto-viscoplastic Formulation based on Fast Fourier Transforms for the Prediction of Micromechanical Fields in Polycrystalline materials, International Journal of Plasticity 32 - 33 (2012) $59-69$.

[51] R. deWit, Linear theory of static disclinations, in: J. Simmons, R. deWit, R. Bullough (Eds.), Fundamental Aspects of Dislocation Theory, vol. 1, National Bureau of Standards Special Publication 317, Washington D.C., 651-680, 1970.

[52] R. deWit, Theory of Disclinations: II. Continuous and Discrete Disclinations in Anisotropic Elasticity, Journal of Research of the National Bureau of Standards - A. Physics and Chemistry 77A (1973) 49-100. 
[53] R. deWit, Theory of Disclinations: III. Continuous and Discrete Disclinations in Isotropic Elasticity, Journal of Research of the National Bureau of Standards - A. Physics and Chemistry 77A (1973) 359-368.

[54] R. deWit, Theory of Disclinations: IV. Straight Disclinations, Journal of Research of the National Bureau of Standards - A. Physics and Chemistry 77A (1973) 607-658.

[55] J. Nye, Some Geometrical Relations in Dislocated Crystals, Acta Metallurgica 1 (1953) 153-162.

[56] R. D. Mindlin, H. F. Tiersten, Effects of couple stresses in linear elasticity, Archive for rational mechanics and analysis 11 (1962) 415-448.

[57] M. V. Upadhyay, L. Capolungo, V. Taupin, C. Fressengeas, Elastic Constitutive Laws for Incompatible Crystalline Media: the Contributions of Dislocations, Disclinations and G-disclinations, Philosophical Magazine 93 (2013) 794-832.

[58] R. A. Toupin, Elatic materials with couple stresses, Archive for rational mechanics and analysis 11 (1962) 385-414.

[59] E. Kröner, On the physical reality of torque stresses in continuum mechanics, International Journal of Engineering Science 1 (1963) 261-278.

[60] R. Maranganti, P. Sharma, A Novel Atomistic Approach to Determine Strain-Gradient Elasticity Constants: Tabulation and Comparison for Various Metals, Semiconductors, Silica, Polymers and the (ir) Relevance for Nanotechnologies, Journal of the Mechanics and Physics of Solids 55 (2007) $1823-1852$.

[61] D. Seif, G. Po, M. Mrovec, M. Lazar, C. Elsässer, P. Gumbsch, Atomistically enabled nonsingular anisotropic elastic representation of near-core dislocation stress fields in $\alpha$-iron, Physical Review B 91 (2015) 184102.

[62] C. Fressengeas, V. Taupin, L. Capolungo, An elasto-plastic theory of dislocation and disclination fields, International Journal of Solids and Structures 48 (2011) 3499-3509.

[63] S. Forest, G. Cailletaud, R. Sievert, A Cosserat theory for elastoviscoplastic single crystals at finite deformation, Archives of Mechanics 4 (1997) 705-736. 
[64] J. Mayeur, D. L. McDowell, D. J. Bammann, Dislocation-based micropolar single crystal plasticity: Comparison of multi- and single criterion theories, Journal of the Mechanics and Physics of Solids 59 (2011) 398422 .

[65] J. Michel, H. Moulinec, P. Suquet, A Computational Method based on Augmented Lagrangians and Fast Fourier Transforms for Composites with High Contrast, Computer Modelling in Engineering \& Sciences 1 (2000) $79-88$.

[66] J. Michel, H. Moulinec, P. Suquet, A Computational Scheme for Linear and Non-Linear Composites with Arbitrary Phase Contrast, International Journal for Numerical Methods in Engineering 52 (2001) 139 160.

[67] L. Dellinger, D. Vasicek, C. Sondergeld, Kelvin Notation for Stabilizing Elastic Constant Inversion, Revue de l'Institute Francaise de Petrol 53 (1998) $709-719$.

[68] W. Müller, Fourier transforms and their application to the formation of textures and changes of morphology in solids, in: IUTAM Symposium on Transformation Problems in Composite and Active Materials, Kluwer Academic Publishers, 61-72, 1998.

[69] W. Press, S. Teukolsky, W. Vetterling, B. Flannery, Numerical recipies in $\mathrm{C}++$, Cambridge University Press, USA, 2002.

[70] S. Berbenni, V. Taupin, K. Djaka, C. Fressengeas, A Numerical Spectral Approach for Solving Elasto-Static Field Dislocation and G-Disclination Mechanics, International Journal of Solids and Structures 51 (2014) $4157-4175$.

[71] R. A. Lebensohn, A. Needleman, Numerical implementation of nonlocal polycrystal plasticity using Fast Fourier Transforms, Journal of the Mechanics and Physics of Solids Submitted.

[72] W. Pantleon, Resolving the geometrically necessary dislocation content by conventional electron backscattering diffraction, Scripta Materialia 58 (2008) 994-997. 
[73] B. Beausir, C. Fressengeas, Disclination Densities from EBSD Orientation Mapping, International Journal of Solids and Structures 50 (2013) $137-146$.

[74] C. Landon, B. Adams, J. Kacher, High-Resolution Methods for Characterizing Mesoscale Dislocation Structures, Journal of Engineering Materials and Technology 130 (2008) 021004.

[75] S. Birosca, F. Di Gioacchino, S. Stekovic, M. Hardy, A quantitative approach to study the effect of local texture and heterogeneous plastic strain on the deformation micromechanism in RR1000 nickel-based superalloy, Acta Materialia 74 (2014) 110-124.

[76] E. Rauch, M. Véron, Automated crystal orientation and phase mapping in TEM, Materials Characterization 98 (2014) $1-9$.

[77] G. Simmons, H. Wang, Single Crystal Elastic Constants and Calculated Aggregate Properties: A Handbook, MIT press, Cambridge, 1971.

[78] L. Li, P. Anderson, M.-G. Lee, E. Bitzek, P. Derlet, H. Van Swygenhoven, The stress-strain response of nanocrystalline metals: a quantized crystal plasticity approach, Acta Materialia 57 (2009) 812-822.

[79] S. Van Petegem, L. Li, P. Anderson, H. Van Swygenhoven, Deformation mechanisms in nanocrystalline materials: Insights from in-situ diffraction and crystal plasticity modeling, Thin Solid Films 530 (2013) 20-24.

[80] E. Bringa, A. Caro, Y. Wang, M. Victoria, J. McNaney, B. Remington, R. Smith, B. Torralva, H. Van Swygenhoven, Ultrahigh strength in nanocrystalline materials under shock loading, Science 309 (2005) $1838-1841$.

[81] F. C. Frank, The Resultant Content of Dislocations in an Arbitrary Intercrystalline Boundary, in: Symposium on The Plastic Deformation of Crystalline Solids, Mellon Institute, Pittsburg, 150, 1950.

[82] B. Bilby, R. Bullough, E. Smith, Continuous Distributions of Dislocations: A new Application of the Methods of Non-Riemannian Geometry, Proceedings of the Royal Society of London: Series A, Mathematical and physical sciences 231 (1955) $263-273$. 
[83] J. C. M. Li, Disclination Model of High Angle Grain Boundaries, Surface Science 31 (1972) 12-26.

[84] S. Forest, D. K. Trinh, Generalized continua and non-homogeneous boundary conditions in homogenization methods, Zeitschrift für Angewandte Mathematik und Mechanik 91 (2011) 90 - 109.

[85] M. Murayama, J. M. Howe, H. Hidaka, S. Takaki, Atomic-Level Observation of Disclination Dipoles in Mechanically Milled Nanocrystalline Fe, Science 295 (2002) 2433 - 2435.

[86] A. Acharya, J. Bassani, Incompatibility and Crystal Plasticity, Journal of the Mechanics and Physics of Solids 48 (2000) 1565 - 1595.

[87] S. Forest, F. Barbe, G. Cailletaud, Cosserat modelling of size effects in the mechanical behavior of polycrystals and multi-phase materials, International Journal of Solids and Structures 37 (2000) $7105-7126$.

[88] M. E. Gurtin, A Gradient Theory of Single Crystal Viscoplasticity that Accounts for Geometrically Necessary Dislocations, Journal of the Mechanics and Physics of Solids 50 (2002) 5-32.

[89] J. Clayton, D. L. McDowell, D. J. Bammann, Modeling dislocations and disclinations with finite micropolar elasticity, International Journal of Plasticity 22 (2006) 210-256.

[90] A. Ma, F. Roters, D. Raabe, On the consideration of interactions between dislocations and grain boundaries in crystal plasticity finite element modeling theory, experiments, and simulations, Acta Materialia 54 (2006) 2181-2194.

[91] J. Gerken, Formulation and solution of a crystal plasticity constitutive model with slip gradient effects, $\mathrm{PhD}$ dissertation, Cornell Univeristy, Department of Mechanical Engineering, 2007.

[92] A. Acharya, F. Fressengeas, Coupled phase transformations and plasticity as a field theory of deformation incompatibility 174 (2012) 87-94.

[93] R. Desmorat, Décomposition de Kelvin et concept de contraintes effectives multiples pour les matériaux anisotropes, Comptes Rendus Mécanique 337 (2009) 733 - 738. 
[94] R. Marull, R. Desmorat, Kelvin Modes Based Cubic Plasticity and Induced Anisotropic Damage: Application to Creep of AM1 Single Crystal, in: H. Altenbach, S. Kruch (Eds.), Advanced Materials Modelling for Structures, vol. 19, Springer Berlin Heidelberg, 217-227, 2013.

[95] W. H. Press, S. A. Teukolsky, W. T. Vetterling, B. P. Flannery, Numerical Recipes in Fortran 77: The Art of Scientific Computing, vol. 1, Press Syndicate of the University of Cambridge, Cambridge, 1997.

[96] M. V. Upadhyay, On the role of defect incompatibilities on mechanical properties of polycrystalline aggregates: a multi-scale study, PhD dissertation, Georgia Institute of Technology, Department of Mechanical Engineering, 2014.

\section{Appendix A. Kelvin decomposition of an elasticity tensor}

In this section, the basis tensors obtained from the Kelvin decomposition of the elasticity tensor $\boldsymbol{A}$ is developed. The original methodology for the classical elastic framework is proposed in the work of Desmorat and Marull $[93,94]$, to which the interested readers are directed. In the following, the superscripts $D$ and $e$ for the deviatoric couple stress and elastic curvatures tensor are dropped.

Appendix A.1. Kelvin decomposition in couple stress theory

The couple stress elastic constitutive law relates the generally asymmetric and deviatoric couple stresses and elastic curvatures. Therefore, the equivalent Kelvin and Voigt vector and tensor representations will have 9 components, in lieu of 6 for the classical elasticity [93] case.

The elasticity fourth rank tensor $\boldsymbol{A}$ (the corresponding Voigt matrix representation is $[A])$ has eigenvalues $\Phi^{(I)}$ and corresponding second rank symmetric eigentensors $e^{(I)}$ (with eigenvectors $\hat{e}^{(I)}$ ) solutions of the eigenproblem

$$
\begin{aligned}
\boldsymbol{A}: \boldsymbol{e}^{(I)}=\Phi^{(I)} \boldsymbol{e}^{(I)}, & \boldsymbol{e}^{(I)}: \boldsymbol{e}^{(J)} & =\delta_{I J} \\
{[A] \hat{e}^{(I)}=\Phi^{(I)} \hat{e}^{(I)}, } & \hat{e}^{(I)} \cdot \hat{e}^{(J)} & =\delta_{I J}
\end{aligned}
$$

The eigenvalues $\Phi^{(I)}$ can at most attain nine different values. The extended Kelvin decomposition of the elasticity tensor $\boldsymbol{A}$ is then given as

$$
\boldsymbol{A}=\sum_{I=1}^{9} \Phi^{(I)} \boldsymbol{e}^{(I)} \otimes \boldsymbol{e}^{(I)} \quad \Leftrightarrow \quad[A]=\sum_{I=1}^{9} \Phi^{(I)} \hat{e}^{(I)} \otimes\left(\hat{e}^{(I)}\right)^{T}
$$


While a family of nine orthogonal eigentensors $\boldsymbol{e}^{(I)}$ is always existing, some of the eigenvalues can be repeated - making the family of eigentensors, non-unique - depending on the material symmetry. The terms of identical moduli $\Phi^{(I)}=\Phi^{K}$ can be conveniently grouped as,

$$
\boldsymbol{A}=\sum_{K=1}^{N \leq 9} \Phi^{K} \boldsymbol{P}^{K} \quad \boldsymbol{Q}^{K}=\sum_{I / \Phi^{(I)}=\Phi^{K}} \boldsymbol{e}^{(I)} \otimes \boldsymbol{e}^{(I)}
$$

The projectors $\boldsymbol{Q}^{K}$ are unique for a given elasticity tensor $\boldsymbol{A}$ and they naturally lead to the definition of Kelvin couple stresses,

$$
\boldsymbol{M}^{K}=\boldsymbol{Q}^{K}: \boldsymbol{M}
$$

For a given material symmetry, the above equation defines the Kelvin couple stress $\boldsymbol{M}^{K}$ as the projection of the couple stress tensor on the $K^{\text {th }}$ Kelvin mode in a unique and objective manner. If the same projection is made for the elastic curvature, i.e. $\boldsymbol{\kappa}^{K}=\boldsymbol{Q}^{K}$ : $\boldsymbol{\epsilon}$, the elasticity law $\boldsymbol{M}=$ $\boldsymbol{A}: \boldsymbol{\kappa}$ is equivalent to

$$
\boldsymbol{M}^{K}=\Phi^{K} \boldsymbol{Q}^{K} \quad \forall K
$$

Appendix A.2. Kelvin decomposition for isotropic symmetry

Due to limited knowledge on the symmetries of the elasticity tensor $\boldsymbol{A}$, the eigenvectors and tensors related to the couple stresses and elastic curvatures are developed in the case of isotropic symmetry.

Let the couple stresses and elastic curvatures be written in the Voigt notation as follows:

$$
\begin{array}{r}
\hat{M}=\left(M_{x x}, M_{y y}, M_{z z}, M_{y z}, M_{x z}, M_{x y}, M_{z y}, M_{z x}, M_{y x}\right)^{T} \\
\hat{\kappa}=\left(\kappa_{x x}, \kappa_{y y}, \kappa_{z z}, \kappa_{y z}, \kappa_{x z}, \kappa_{x y}, \kappa_{z y}, \kappa_{z x}, \kappa_{y x}\right)^{T}
\end{array}
$$

the elasticity law $\boldsymbol{M}=\boldsymbol{A}: \boldsymbol{\kappa}$ takes the isotropic form (in the natural anisotropy basis),

$$
\hat{M}=[A] \hat{\kappa} \quad[A]^{-1}=\left[\begin{array}{ccccccccc}
A_{1} & 0 & 0 & 0 & 0 & 0 & 0 & 0 & 0 \\
0 & A_{1} & 0 & 0 & 0 & 0 & 0 & 0 & 0 \\
0 & 0 & A_{1} & 0 & 0 & 0 & 0 & 0 & 0 \\
0 & 0 & 0 & A_{1} & 0 & 0 & 0 & 0 & 0 \\
0 & 0 & 0 & 0 & A_{1} & 0 & 0 & 0 & 0 \\
0 & 0 & 0 & 0 & 0 & A_{1} & 0 & 0 & 0 \\
0 & 0 & 0 & 0 & 0 & 0 & A_{1} & 0 & 0 \\
0 & 0 & 0 & 0 & 0 & 0 & 0 & A_{1} & 0 \\
0 & 0 & 0 & 0 & 0 & 0 & 0 & 0 & A_{1}
\end{array}\right]_{\left(\overrightarrow{\boldsymbol{b}}_{1}, \overrightarrow{\boldsymbol{b}}_{2}, \overrightarrow{\vec{b}}_{3}\right)}
$$


here $A_{2}$ is assumed to be zero in the isotropic case. Also note that both couple stress and elastic curvature in the constitutive relationship are deviatoric in nature, therefore the above form of the tensor automatically deals with these. There is only one non-zero Kelvin moduli,

$\Phi^{K=1}=\Phi^{(1)}=\Phi^{(2)}=\Phi^{(3)}=\Phi^{(4)}=\Phi^{(5)}=\Phi^{(6)}=\Phi^{(7)}=\Phi^{(8)}=\Phi^{(9)}=A_{1}($

The 9 corresponding eigentensors in the basis $\left(\overrightarrow{\boldsymbol{b}}_{1}, \overrightarrow{\boldsymbol{b}}_{2}, \overrightarrow{\boldsymbol{b}}_{3}\right)$ are

$$
\begin{aligned}
& \boldsymbol{e}^{(1)}=\frac{1}{\sqrt{3}} \mathbf{I}, \quad \boldsymbol{e}^{(2)}=\frac{\overrightarrow{\boldsymbol{b}}_{1} \otimes \overrightarrow{\boldsymbol{b}}_{1}-\overrightarrow{\boldsymbol{b}}_{2} \otimes \overrightarrow{\boldsymbol{b}}_{2}}{\sqrt{2}}, \quad \boldsymbol{e}^{(3)}=\frac{\overrightarrow{\boldsymbol{b}}_{1} \otimes \overrightarrow{\boldsymbol{b}}_{1}+\overrightarrow{\boldsymbol{b}}_{2} \otimes \overrightarrow{\boldsymbol{b}}_{2}-2 \overrightarrow{\boldsymbol{b}}_{3} \otimes \overrightarrow{\boldsymbol{b}}_{3}}{\sqrt{6}} \\
& \boldsymbol{e}^{(4)}=\overrightarrow{\boldsymbol{b}}_{2} \otimes \overrightarrow{\boldsymbol{b}}_{3}, \quad \boldsymbol{e}^{(5)}=\overrightarrow{\boldsymbol{b}}_{1} \otimes \overrightarrow{\boldsymbol{b}}_{3}, \quad \boldsymbol{e}^{(6)}=\overrightarrow{\boldsymbol{b}}_{1} \otimes \overrightarrow{\boldsymbol{b}}_{2}, \\
& \boldsymbol{e}^{(7)}=\overrightarrow{\boldsymbol{b}}_{3} \otimes \overrightarrow{\boldsymbol{b}}_{2}, \quad \boldsymbol{e}^{(8)}=\overrightarrow{\boldsymbol{b}}_{3} \otimes \overrightarrow{\boldsymbol{b}}_{1}, \quad \boldsymbol{e}^{(9)}=\overrightarrow{\boldsymbol{b}}_{2} \otimes \overrightarrow{\boldsymbol{b}}_{1}
\end{aligned}
$$

These can be represented in tensorial forms using nine basis $B_{2}$ tensors. In the following these tensors are renamed such that the first 8 tensors upon performing an inner product with the couple stress tensor, extract its deviatoric component and the ninth one extracts it hydrostatic component (which is zero) as,

$$
\begin{aligned}
& B_{2}^{(1)}=\frac{1}{\sqrt{2}}\left[\begin{array}{ccc}
-1 & 0 & 0 \\
0 & 1 & 0 \\
0 & 0 & 0
\end{array}\right] ; B_{2}^{(2)}=\frac{1}{\sqrt{6}}\left[\begin{array}{ccc}
-1 & 0 & 0 \\
0 & -1 & 0 \\
0 & 0 & 2
\end{array}\right] ; B_{2}^{(3)}=\left[\begin{array}{lll}
0 & 0 & 0 \\
0 & 0 & 1 \\
0 & 0 & 0
\end{array}\right] ; \\
& B_{2}^{(4)}=\left[\begin{array}{lll}
0 & 0 & 1 \\
0 & 0 & 0 \\
0 & 0 & 0
\end{array}\right] ; B_{2}^{(5)}=\left[\begin{array}{ccc}
0 & 1 & 0 \\
0 & 0 & 0 \\
0 & 0 & 0
\end{array}\right] ; B_{2}^{(6)}=\left[\begin{array}{ccc}
0 & 0 & 0 \\
0 & 0 & 0 \\
0 & 1 & 0
\end{array}\right] ; \\
& B_{2}^{(7)}=\left[\begin{array}{lll}
0 & 0 & 0 \\
0 & 0 & 0 \\
1 & 0 & 0
\end{array}\right] ; B_{2}^{(8)}=\left[\begin{array}{lll}
0 & 0 & 0 \\
1 & 0 & 0 \\
0 & 0 & 0
\end{array}\right] ; B_{2}^{(9)}=\frac{1}{\sqrt{3}}\left[\begin{array}{ccc}
1 & 0 & 0 \\
0 & 1 & 0 \\
0 & 0 & 1
\end{array}\right]
\end{aligned}
$$

The projectors can be defined as

$$
\begin{aligned}
\boldsymbol{Q}^{K=1} & =\boldsymbol{e}^{(1)} \otimes \boldsymbol{e}^{(1)} \\
\boldsymbol{Q}^{K=2} & =\boldsymbol{e}^{(2)} \otimes \boldsymbol{e}^{(2)}+\boldsymbol{e}^{(3)} \otimes \boldsymbol{e}^{(3)} \\
\boldsymbol{Q}^{K=3} & =\boldsymbol{e}^{(4)} \otimes \boldsymbol{e}^{(4)}+\boldsymbol{e}^{(5)} \otimes \boldsymbol{e}^{(5)}+\boldsymbol{e}^{(6)} \otimes \boldsymbol{e}^{(6)} \\
& +\boldsymbol{e}^{(7)} \otimes \boldsymbol{e}^{(7)}+\boldsymbol{e}^{(8)} \otimes \boldsymbol{e}^{(8)}+\boldsymbol{e}^{(9)} \otimes \boldsymbol{e}^{(9)}
\end{aligned}
$$

The Kelvin stresses $\boldsymbol{M}^{K}=\boldsymbol{Q}^{K}: \boldsymbol{M}$ are then obtained as: 
1) $\boldsymbol{M}^{K=1}=\boldsymbol{M}^{H}$ is the hydrostatic component of the deviatoric couple stress and is therefore equal to zero.

2) $\boldsymbol{M}^{K=2}=\boldsymbol{M}^{d}$ is the diagonal part of the deviatoric couple stress in natural anisotropy basis and is associated to the Kelvin modulus $A_{1}$.

3) $\boldsymbol{M}^{K=3}=\boldsymbol{M}^{\bar{d}}$ is the off-diagonal deviatoric couple stress in this same basis and is also associated to the Kelvin modulus $A_{1}$.

The two deviatoric tensors are then naturally obtained from the Kelvin analysis as

$$
\boldsymbol{M}=\boldsymbol{M}^{D}=\boldsymbol{M}^{d}+\boldsymbol{M}^{\bar{d}}
$$

It should be noted that while the Kelvin moduli (eigenvalues) are dependent on the type of elastic anisotropy, the basis tensors remain same irrespective of the anisotropy.

\section{Appendix B. Discrete Fourier transforms}

In this section, a 3-dimensional DFT based framework is proposed to overcome the Gibbs phenomenon. This work is a generalization of the existing 2-dimensional implementations of the approach [68-70]. The cubic microstrcture is discretized into a regular grid of $N_{1} \times N_{2} \times N_{3}$ voxels with periodic boundary conditions along all the surfaces. The position vectors are defined as $\overrightarrow{\boldsymbol{x}}=\left((l-1) \delta_{1},(m-1) \delta_{2},(n-1) \delta_{3}\right)$, where $l=1 \rightarrow N_{1}, m=$ $1 \rightarrow N_{2}, n=1 \rightarrow N_{3}$ and $\delta_{1}, \delta_{2}$ and $\delta_{3}$ are the voxel sizes in directions 1,2 and 3 respectively. The total number of voxels are $N_{\text {tot }}=N_{1} N_{2} N_{3}$.

In the following, derivations are performed adopting the convention used by "fourn" FORTRAN subroutine [95]. In the convention adopted by the book, DFTs are defined as:

$$
\begin{aligned}
& \hat{f}\left(\xi_{1}, \xi_{2}, \xi_{3}\right)= \\
& \sum_{l=1}^{N_{1}} \sum_{m=1}^{N_{2}} \sum_{n=1}^{N_{3}} f(i, j, k) e^{2 \pi i(l-1)\left(\xi_{1}-1\right) / N_{1}} e^{2 \pi i(m-1)\left(\xi_{2}-1\right) / N_{2}} e^{2 \pi i(n-1)\left(\xi_{3}-1\right) / N_{3}}
\end{aligned}
$$

and

$$
\begin{aligned}
& f(l, m, n)= \\
& \sum_{l=1}^{N_{1}} \sum_{m=1}^{N_{2}} \sum_{n=1}^{N_{3}} \frac{\hat{f}\left(\xi_{1}, \xi_{2}, \xi_{3}\right) e^{-2 \pi i(l-1)\left(\xi_{1}-1\right) / N_{1}} e^{-2 \pi i(m-1)\left(\xi_{2}-1\right) / N_{2}} e^{-2 \pi i(n-1)\left(\xi_{3}-1\right) / N_{3}}}{N_{\text {tot }}}
\end{aligned}
$$


where $i=\sqrt{-1} . \xi_{1}, \xi_{2}$ and $\xi_{3}$ are the frequencies corresponding to $l, m$ and $n$, respectively.

Using the shift theorem for Fourier transforms gives:

$$
\begin{aligned}
& F T(f(l+P, m+Q, n+R))= \\
& e^{-2 \pi i P \xi_{1} / N_{1}} e^{-2 \pi i Q \xi_{2} / N_{2}} e^{-2 \pi i R \xi_{3} / N_{3}} F T(f(l, m, n))
\end{aligned}
$$

where FT represents the Fourier transform operator and $P, Q, R$ are some integers.

Also, the partial derivative in the adopted convention is given as:

$$
F T\left(\frac{\partial^{3} f(l, m, n)}{\partial x_{1} \partial x_{2} \partial x_{3}}\right)=\left(-i k_{1}\right)\left(-i k_{2}\right)\left(-i k_{3}\right) F T(f(l, m, n))
$$

where $k_{1}=2 \pi \xi_{1}, k_{2}=2 \pi \xi_{2}$ and $k_{3}=2 \pi \xi_{3}$ are the angular frequencies.

Appendix B.1. Differentiation rules

Let $\partial^{a+b+c} f(l, m, n) / \partial x_{1}^{a} \partial x_{2}^{b} \partial x_{3}^{c}$ correspond to the $a$ th, $b$ th and $c$ th partial derivative of a funtion $f(l, m, n)$ with respect to $x_{1}, x_{2}$ and $x_{3}$, respectively. In the present work, only the partial dervatives corresponding to $a+b+c=$ 1, 2, 3 and 4 are considered. The first and second order partial derivatives are computed on the discrete grid based on the 27 -voxel finite difference approximation of partial derivatives using centered differences. The accuracy achieved is of the order $O\left(\delta_{1}^{2}, \delta_{2}^{2}, \delta_{3}^{2}\right)$.

Appendix B.1.1. First order partial derivatives

The continuous Fourier transforms of first order partial derivatives are the above equations are $-i k_{1},-i k_{2}$ and $-i k_{3}$. Approximating the first order partial derivatives using the central difference approach, their equivalent discrete Fourier transforms are given as:

$$
\begin{aligned}
& -i k_{1} \leftrightarrow \frac{e^{-2 \pi i\left(\xi_{1}-1\right) / N_{1}}-e^{2 \pi i\left(\xi_{1}-1\right) / N_{1}}}{2 \delta_{1}}=-\frac{i}{\delta_{1}} \sin \left(\frac{2 \pi\left(\xi_{1}-1\right)}{N_{1}}\right) \\
& -i k_{2} \leftrightarrow \frac{e^{-2 \pi i\left(\xi_{2}-1\right) / N_{2}}-e^{2 \pi i\left(\xi_{2}-1\right) / N_{2}}}{2 \delta_{2}}=-\frac{i}{\delta_{2}} \sin \left(\frac{2 \pi\left(\xi_{2}-1\right)}{N_{2}}\right) \\
& -i k_{3} \leftrightarrow \frac{e^{-2 \pi i\left(\xi_{3}-1\right) / N_{3}}-e^{2 \pi i\left(\xi_{3}-1\right) / N_{3}}}{2 \delta_{3}}=-\frac{i}{\delta_{3}} \sin \left(\frac{2 \pi\left(\xi_{3}-1\right)}{N_{3}}\right)
\end{aligned}
$$


Appendix B.1.2. Second order partial derivatives

Similarly, approximating the second order partial derivatives using the central difference scheme, the continuous Fourier transform and the corresponding discrete Fourier transform are:

$$
\begin{aligned}
-k_{1}^{2} & \leftrightarrow \frac{2}{\delta_{1}^{2}}\left(1-\cos \left(\frac{2 \pi\left(\xi_{1}-1\right)}{N_{1}}\right)\right) \\
-k_{2}^{2} & \leftrightarrow \frac{2}{\delta_{2}^{2}}\left(1-\cos \left(\frac{2 \pi\left(\xi_{2}-1\right)}{N_{2}}\right)\right) \\
-k_{3}^{2} & \leftrightarrow \frac{2}{\delta_{3}^{2}}\left(1-\cos \left(\frac{2 \pi\left(\xi_{3}-1\right)}{N_{3}}\right)\right) \\
-k_{1} k_{2} & \leftrightarrow-\frac{1}{\delta_{1} \delta_{2}} \sin \left(\frac{2 \pi\left(\xi_{1}-1\right)}{N_{1}}\right) \sin \left(\frac{2 \pi\left(\xi_{2}-1\right)}{N_{2}}\right) \\
-k_{1} k_{3} & \leftrightarrow-\frac{1}{\delta_{1} \delta_{3}} \sin \left(\frac{2 \pi\left(\xi_{1}-1\right)}{N_{1}}\right) \sin \left(\frac{2 \pi\left(\xi_{3}-1\right)}{N_{3}}\right) \\
-k_{2} k_{3} & \leftrightarrow-\frac{1}{\delta_{2} \delta_{3}} \sin \left(\frac{2 \pi\left(\xi_{2}-1\right)}{N_{2}}\right) \sin \left(\frac{2 \pi\left(\xi_{3}-1\right)}{N_{3}}\right)
\end{aligned}
$$

Formulae for third and fourth order derivatives, along with a generic

formula for $n^{\text {th }}$ order derivative have also been derived elsewhere [96] but are not presented here for the sake of brevity.

\section{Appendix C. Algorithm}

Figure C.15 shows the algorithm for the CSEVP DFT-I procedure described in section 4.2 


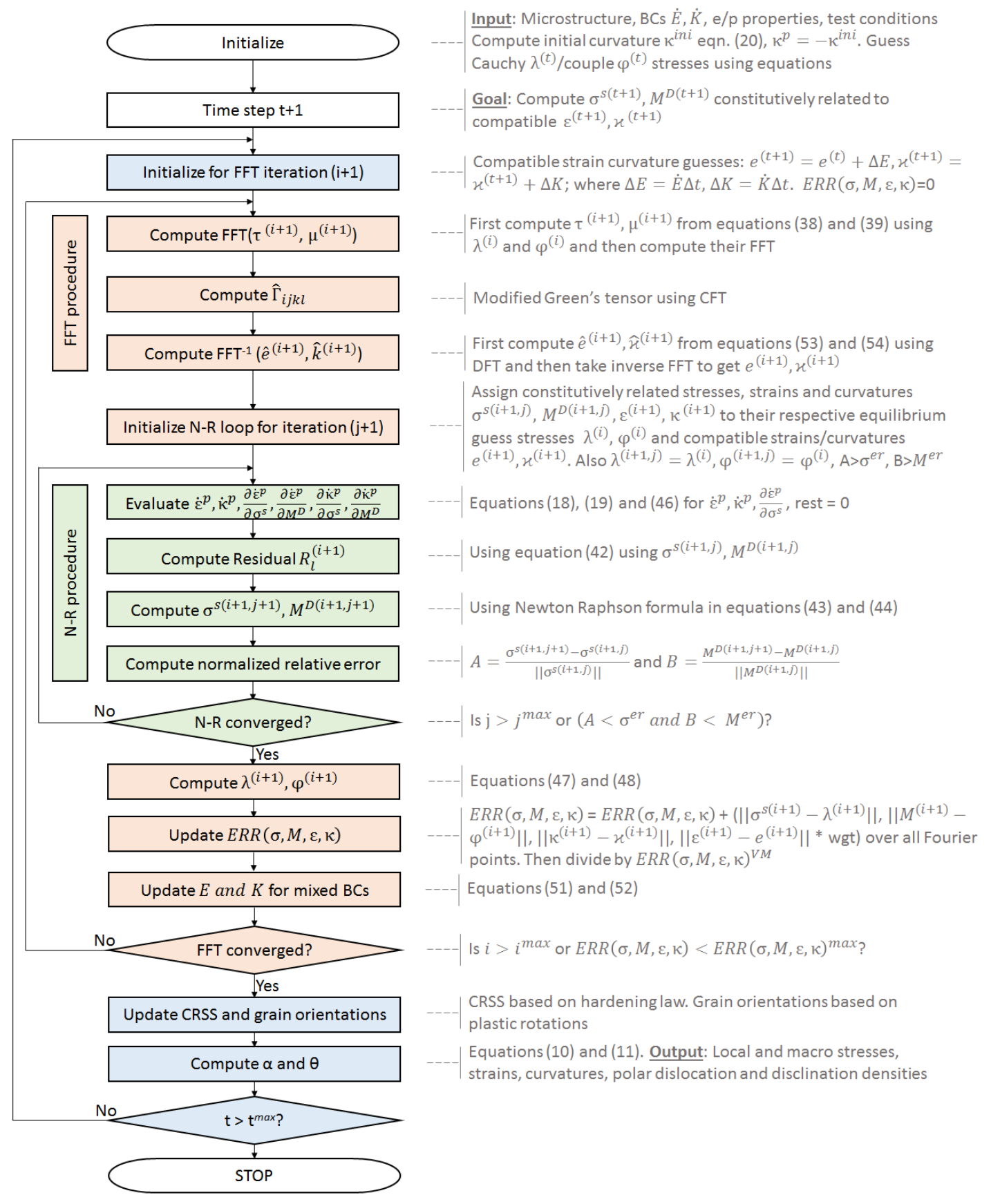

Figure C.15: Algorithm for CSEVP DFT-I. 